\title{
The Stellar CME-Flare Relation: What Do Historic Observations Reveal?
}

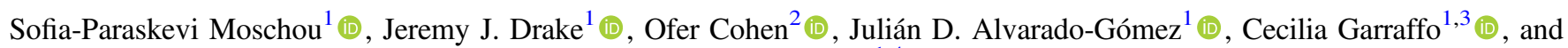 \\ Federico Fraschetti ${ }^{1,4}$ (i) \\ ${ }^{1}$ Center for Astrophysics | Harvard \& Smithsonian, 60 Garden Street, Cambridge, MA 02138, USA \\ ${ }^{2}$ Lowell Center for Space Science and Technology, University of Massachusetts, Lowell, MA, USA \\ ${ }^{3}$ IACS, Harvard University, 33 Oxford Street Cambridge, MA 02138, USA \\ ${ }^{4}$ Dept. of Planetary Sciences-Lunar and Planetary Laboratory, University of Arizona, Tucson, AZ, 85721, USA \\ Received 2019 January 16; revised 2019 March 27; accepted 2019 April 19; published 2019 May 31
}

\begin{abstract}
Solar coronal mass ejections (CMEs) and flares have a statistically well-defined relationship, with more energetic X-ray flares corresponding to faster and more massive CMEs. How this relationship extends to more magnetically active stars is a subject of open research. Here we study the most probable stellar CME candidates associated with flares captured in the literature to date, all of which were observed on magnetically active stars. We use a simple CME model to derive masses and kinetic energies from observed quantities and transform associated flare data to the Geostationary Operational Environmental Satellite $1-8 \AA$ band. Derived CME masses range from $\sim 10^{15}$ to $10^{22} \mathrm{~g}$. Associated flare X-ray energies range from $10^{31}$ to $10^{37} \mathrm{erg}$. Stellar CME masses as a function of associated flare energy generally lie along or below the extrapolated mean for solar events. In contrast, CME kinetic energies lie below the analogous solar extrapolation by roughly 2 orders of magnitude, indicating approximate parity between flare X-ray and CME kinetic energies. These results suggest that the CMEs associated with very energetic flares on active stars are more limited in terms of the ejecta velocity than the ejecta mass, possibly because of the restraining influence of strong overlying magnetic fields and stellar wind drag. Lower CME kinetic energies and velocities present a more optimistic scenario for the effects of CME impacts on exoplanets in close proximity to active stellar hosts.
\end{abstract}

Key words: planet-star interactions - stars: activity - stars: flare - stars: late-type - Sun: coronal mass ejections (CMEs) - X-rays: stars

\section{Introduction}

For decades, since the first space-based coronal mass ejection (CME) observations in the 1970s (Tousey et al. 1973), the Sun has been the only star that allowed for direct CME observation. Recently, with the discovery of multiple exoplanetary systems, there is an increasing scientific interest in determining the effects of stellar activity on planetary atmospheres and habitability (e.g., Khodachenko et al. $2007 \mathrm{a}, 2007 \mathrm{~b}$ ). These efforts are similar in some ways to what has been done up to now in the field of space weather (Kahler 2001; Zhang et al. 2007; Webb et al. 2009; Yashiro \& Gopalswamy 2009; Cane et al. 2010; Vourlidas et al. 2011; Cliver \& Dietrich 2013; Reames 2013; Gopalswamy 2016). While space weather in the solar environment is comparatively better understood, the vast majority of the stars in our Galaxy are red dwarfs, and only a small percentage are Sun-like stars. The magnetic behavior of stars quite different from the Sun remains uncertain in detail, and it has become imperative that the role of stellar CMEs is assessed in this context on other stars (e.g., Kay et al. 2016).

Solar CMEs and flares are more tightly associated with each other with increasing flaring energy (e.g., Yashiro \& Gopalswamy 2009; Aarnio et al. 2011), with the association reaching a "1-1" ratio for high energies. Solar flares are classified morphologically as either compact with a small number of magnetic loops flaring up for a few minutes or "tworibbon" flares that unwind over longer timescales on the order of hours (see, e.g., Pallavicini et al. 1977; Shibata \& Magara 2011). Two-ribbon flares are associated with an arcade group with complex topology and footpoints that form two parallel chromospheric ribbons visible in $\mathrm{H}_{\alpha}$. Very large solar flares belong in the two-ribbon class, which involves a continuous reconnection starting at the top of the magnetic arcade system and propagating upward toward loops positioned on top of each other flaring serially.

A plethora of stellar flares have been observed in radio, optical, UV, and X-ray wavelengths in active and Sun-like stars and in both single and binary star systems (e.g., Osten et al. 2005; Huenemoerder et al. 2010; Kretzschmar 2011; Notsu et al. 2016; Crosley \& Osten 2018a). All classes of late-type stars are known to flare in soft X-rays (e.g., Schmitt 1994). Pandey \& Singh (2008) showed that (a) late-type G-K dwarfs flare frequently, (b) their flares resemble the two-ribbon solar ones, and (c) even though they are as energetic as $\mathbf{M}$ dwarf flares, they are energetically weaker than flares from pre-mainsequence, giant, and dMe stars. Giant flares from binary systems and young stars with a disk could result from magnetic coupling between the binary members and the young star and its disk, respectively (Graffagnino et al. 1995; Grosso et al. 1997; Tsuboi et al. 2000). As argued in Drake et al. (2013), strong winds of active stars are potentially dominated by CMEs as a result of their extreme flaring activity, with great implications for the energy budget of the system (see also Aarnio et al. 2012; Osten \& Wolk 2015). Later on, Odert et al. (2017) developed a model for estimating mass-loss rates due to CMEs in other stars using the solar flare-CME relations and stellar flaring rates. The authors suggested that solar extrapolations present limitations in their applicability to the young-star regime as they reached CME-driven mass-loss rates higher than the total observationally determined values. In a different approach, Cranmer (2017) exploited the correlations between solar surface-averaged magnetic flux values and mean kinetic 
energy flux in CMEs and the wind to predict CME and wind mass-loss rates in other stars. The main result therein was that the mass-loss rate for stars younger than $1 \mathrm{Gyr}$ is dominated by CMEs. More recently, Vida et al. (2019) presented a large statistical analysis of $\sim 500$ stellar events with line asymmetries in Doppler-shift observations. They measured speeds of the order of $100-300 \mathrm{~km} \mathrm{~s}^{-1}$ and masses of the order of $10^{15}-10^{18} \mathrm{~g}$ and confirmed that cooler stars appear more chromospherically active.

Pallavicini et al. (1990) published a thorough stellar flare survey using EXOSAT that brought to light two stellar flare classes. The first class involved impulsive flares that resemble compact solar flares, while the second class involved flares with longer decay times, which are similar to the two-ribbon solar flare category. The impulsive flares have a short (less than $1 \mathrm{hr}$ ) duration, emit a total X-ray energy on the order of $L_{\mathrm{X}}=10^{30} \mathrm{erg} \mathrm{s}^{-1}$, and are believed to involve a single loop only. The flares with longer decay are 2 orders of magnitude more energetic, $L_{\mathrm{X}}=10^{32} \mathrm{erg} \mathrm{s}^{-1}$; last more than $1 \mathrm{hr}$; and involve an arcade-forming group of loops. As Pandey \& Singh (2012) emphasized, even though there are several similarities between solar and stellar flares, it is difficult to draw a direct parallel, as the latter ones involve orders of magnitude larger energies (Güdel \& Nazé 2009).

It is essential to understand the characteristics of stellar CMEs in order to evaluate the habitability of an exoplanet. Active stars are observed to flare so frequently (e.g., Kashyap et al. 2002; Huenemoerder et al. 2010) that their light curves can oftentimes be approximated by a superposition of flares (e.g., Audard et al. 2000; Caramazza et al. 2007). If a high association rate between stellar CMEs and flares is in place for active stars, then the exoplanets orbiting them will face frequent interactions with transients. This can lead to very high depletion rates, as shown in Cerenkov et al. (2017), but the planetary magnetic field might be able to shield the planetary atmosphere (see, e.g., Cohen et al. 2011).

With current instrumentation, we are unable to directly observe CMEs even in the closest stars; thus, one has to turn to indirect observational evidence for signatures of this eruptive phenomenon. The absorption of emission coming from underlying stellar atmospheric layers by the CME volume is one indirect method that is especially useful for large stellar mass eruptions rather than solar-like ones. Absorption is observed in solar erupting filaments as well (e.g., Subramanian \& Dere 2001; Kundu et al. 2004; Jiang et al. 2006; Vemareddy et al. 2012; Gosain et al. 2016; Chandra et al. 2017), but the CME material does not suffice to cause significant absorption by itself. If the CME-flare association holds for much more energetic events on other stars, we expect considerably more massive CMEs for active stars, and their masses could then provide a valuable observational tool through absorption.

In Section 2, we introduce and briefly explain the Dopplershift and absorption methods currently available for CME tracing on stars other than the Sun. Then, we present all of the known stellar CME candidates identified to date, and in Section 3 we estimate their mass and kinetic energy and place them in the X-ray fluence-CME characteristic property diagram. In Section 4, we present our results, and in Section 5 we discuss sources of discrepancy and other proposed CME detection techniques and mention future missions and computational models that will contribute in the field in the near future. We wrap up this paper with our conclusions in Section 6.

\section{Analysis}

\subsection{Tracking Methods}

Even though stellar flares and superflares (with energies $>10^{33} \mathrm{erg}$; see, e.g., Notsu et al. 2016) are routinely observed in Sun-like and more active stars over a wide range of wavelengths ranging from radio to $\mathrm{X}$-rays, the direct imaging of stellar CMEs is a difficult, if not impossible, task with current instrumentation. For that reason, several observational proxy methods have been proposed to indirectly provide evidence for CME occurrence in other stars (see, e.g., Osten et al. 2017). However, only a handful of possible CME events have been captured for each of two techniques, namely, X-ray continuous absorption (see Moschou et al. 2017) and Doppler shifts in UV wavelengths (Vida et al. 2016).

As explained in Leitzinger et al. (2014), several suspected CMEs have been the focus of observational studies where $\mathrm{X}$-ray absorption in association with energetic flares was seen (Haisch et al. 1983; Ottmann \& Schmitt 1996; Tsuboi et al. 1998; Favata \& Schmitt 1999; Franciosini et al. 2001; Pandey \& Singh 2012), as well as from flare-associated Doppler shifts in Balmer lines (Houdebine et al. 1990; Guenther \& Emerson 1997; Bond et al. 2001; Fuhrmeister \& Schmitt 2004; Leitzinger et al. 2011; Vida et al. 2016). In the next section, we will present all of the CME candidates known from published studies so far based on those two methods.

Continuous X-ray absorption during a stellar flaring event can be used to infer the kinematic characteristics of the obscuring material, e.g., due to a CME or prominence. The best representative of a CME candidate observed through X-ray absorption is the 1997 August 30 Algol event. This was a very large X-ray flare analyzed by Favata \& Schmitt (1999), and the parameters of the associated potential CME were derived by Moschou et al. (2017). The reason that a CME is the most probable scenario for the 1997 August Algol event is that there was sharp increase, by almost 2 orders of magnitude, in the column density, and the continuous absorption in X-rays gradually decayed with an inverse square law with time. As discussed extensively in Moschou et al. (2017), this temporal variation, combined with the lack of rotation modulation of the $\mathrm{X}$-ray signal, is consistent with a CME expanding in a selfsimilar manner away from the stellar surface. Here we examine CME candidates that show a clear column density decay over periods of time of several kiloseconds and treat as prominences events with no substantial temporal decay; see Figure 1. Wherever possible, we will follow a similar analysis technique here as in Moschou et al. (2017) to analyze all of the suspected CMEs inferred through X-ray absorption. It must be noted, however, that it is very difficult to exclude the prominence scenario, even for historic CME candidates with a column density decrease, without resolving the stellar surface. A prominence consisting of dense, cool chromospheric material could undergo complex cooling and heating processes (e.g., Moschou et al. 2015) that affect its ionization degree, and as a result, the prominence could fade when observed in a specific passband (e.g., Ballester et al. 2018).

The observational method based on blueshifted spectral lines involves larger uncertainties because projection effects are difficult to disentangle (e.g., Leitzinger et al. 2011). The 

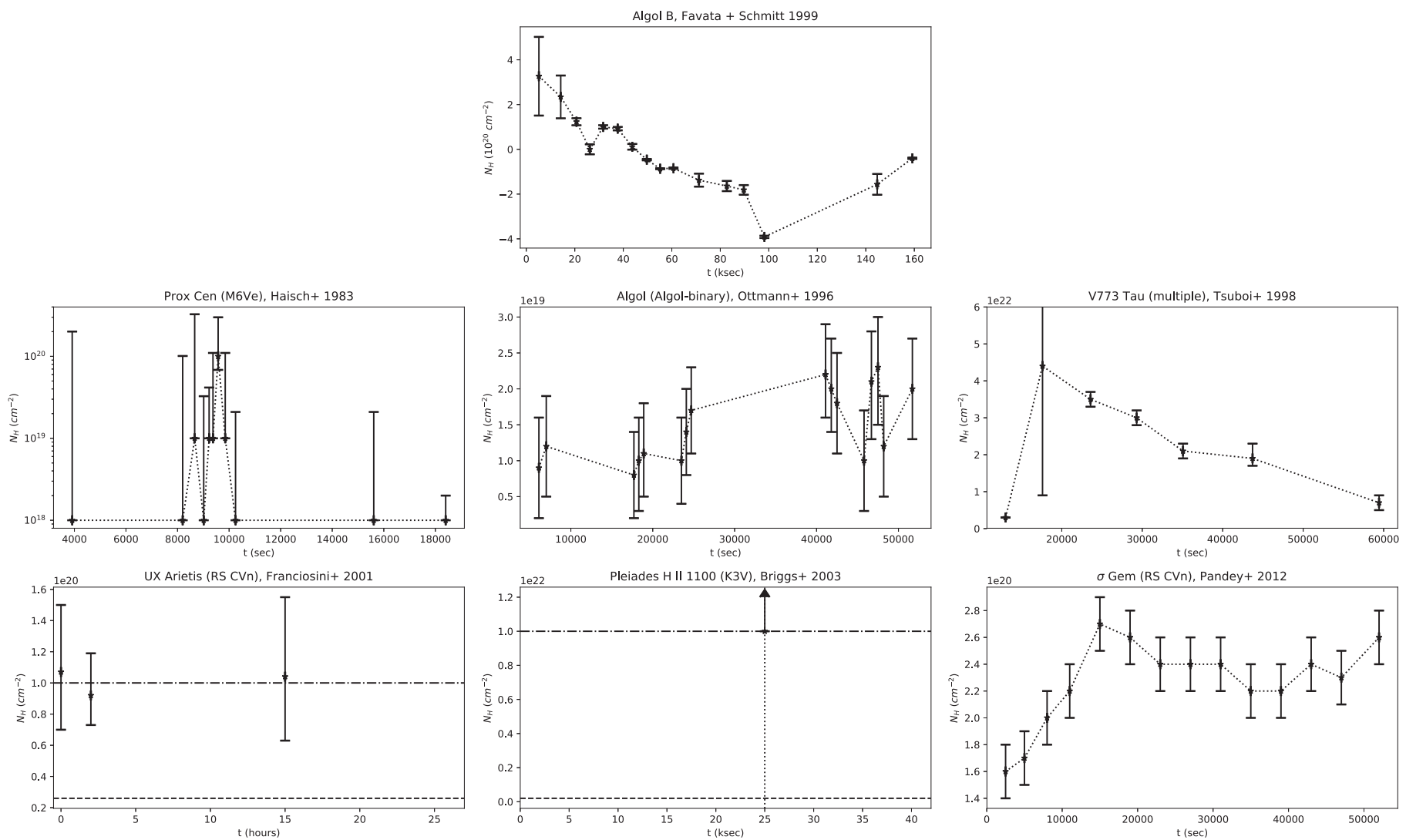

Figure 1. All CME candidates associated with X-ray absorption, including the Algol B event presented in Moschou et al. (2017). The full references and details of each observation can be found in Table 2 . We have offset the observations in time to better capture the evolution of the column density $N_{\mathrm{H}}$, i.e., the rise and decay phases.

inferred velocities in several studies lie in the local chromospheric plasma flow range, i.e., a few tens up to about a couple hundred $\mathrm{km} \mathrm{s}^{-1}$ (Bond et al. 2001; Fuhrmeister \& Schmitt 2004; Leitzinger et al. 2011), making it difficult to distinguish them from other events, such as chromospheric brightenings (Kirk et al. 2017) or chromospheric evaporation (Teriaca et al. 2003; Gupta et al. 2018). Leitzinger et al. (2014) concluded that the CME strength, translated in terms of CME mass or flux, is the most important parameter that controls the efficiency of the Doppler-shift method used to detect them.

In this section, using all historic CME candidates, we will examine the energy regime between the solar events and the Algol extreme flare and associated CME by populating the CME-flare diagram presented in Figure 5 of Moschou et al. (2017). For that, we need a robust unified method to analyze and incorporate all of the historic events so far in that diagram. In short, we use empirical relations to convert optical and UV fluences into X-ray flaring fluences and plot the Doppler-shift CME candidates in the same plot as the X-ray events. We try to keep our assumptions to a minimum and always in the ranges provided in literature when we calculate any quantities, such as CME mass and kinetic energy, that are not provided in the original papers. A summary of all of the analyzable events examined in the current study can be found in Table 3.

\subsection{Events Observed through Doppler Shifts}

In a statistical analysis, Kretzschmar (2011) concluded that for the Sun, the flaring energy on the Geostationary Operational Environmental Satellite (GOES) soft X-ray passband constitutes about $1 \%$ of the total radiated energy.
Furthermore, emission in wavelengths below $50 \mathrm{~nm}$ makes up about $10 \%-20 \%$ of that energy. Finally, the total visible and near-UV parts of the spectrum contain most of the flare energy. It is not straightforward to conclude whether this relation extends to other stars.

Large statistical studies in both Sun-like and more active stars (Butler et al. 1988; Butler 1993; Martínez-Arnáiz et al. 2011) derived empirical relations between $X$-ray luminosity $L_{X}$ and the luminosities of optical lines, such as $\mathrm{H}_{\alpha}$ and $\mathrm{H}_{\gamma}$, i.e., $L_{\mathrm{H} \alpha}$ and $L_{\mathrm{H} \gamma}$, respectively. More specifically, Balmer lines can be converted using the linear relation

$$
L_{X}=32 L_{\mathrm{H} \gamma}=16 L_{\mathrm{H} \alpha}
$$

obtained/verified by multiple space- and ground-based telescopes to soft X-rays integrated in the range (0.04-2.0 keV), as described in Butler et al. (1988) and further extended to a larger range in Butler (1993). Later on, Martínez-Arnáiz et al. (2011) examined a sample of about 300 late-type single stars with spectral types FGKM accounting for basal chromospheric contributions for the first time. From their full sample, they found 243 counterparts with X-ray observations. They showed that there is no universal flux-flux relation for the chromospheric and coronal fluxes, with $\mathrm{dK}$, dKe, and $\mathrm{dMe}^{5}$ stars deviating from the general trend followed by less active stars. More specifically, Martínez-Arnáiz et al. (2011) arrived at the

\footnotetext{
5 The symbol " $\mathrm{d}$ " before the $\mathrm{M}$ and $\mathrm{K}$ stars indicates a subdivision of those late-type dwarf star types, with dMe and dKe being stars with $\mathrm{H}_{\alpha}$ in emission, while $\mathrm{dK}$ and $\mathrm{dM}$ are stars with $\mathrm{H}_{\alpha}$ in absorption.
} 
relationship

$$
\log f_{X}=(-2.19 \pm 0.41)+(1.48 \pm 0.07) \log f_{\mathrm{H}_{\alpha}},
$$

which associates $\mathrm{H}_{\alpha}\left(f_{\mathrm{H}_{\alpha}}\right)$ and X-ray $\left(f_{\mathrm{X}}\right)$ fluxes by fitting the data to a power-law relation (see Figure 5 in Martínez-Arnáiz et al. 2011) using the linear regression presented in Isobe et al. (1990). In other words, Figure 5 in Martínez-Arnáiz et al. (2011) indicates that in the low-energy regime, $F_{\mathrm{H}_{\alpha}}$ and $F_{\mathrm{X}}$ are of the same order of magnitude, but this relation changes as we approach the X-ray saturation regime, with the X-ray flux becoming 1 order of magnitude larger than the $\mathrm{H}_{\alpha}$ flux. The Martínez-Arnáiz et al. (2011) relation (Equation (2)) is more elaborate than the Butler (1993) one (Equation (1)). However, our data set is so inhomogeneous that to avoid further discrepancies, we use the Butler (1993) relation (Equation (1)) to convert the CME candidates that were observed with the Doppler-shift method into X-ray fluxes.

In some of the cases discussed in this paper, the observational passbands are in different wavelengths in the optical/UV regime instead of the $\mathrm{H}_{\alpha}$ or $\mathrm{H}_{\gamma}$ fluxes, which have well-known relations with X-ray emission. The Butler (1993) relation indicates that the X-ray luminosities are typically of the order of a few tens of the optical luminosities observed. For those cases, we multiply the observed optical/UV fluxes by 10 to estimate the X-ray flux inspired by Equation (1) and the high-energy limit of Equation (2). For one case (Katsova et al. 1999), only the broadband luminosity is known. In that case, we divide the broadband luminosity by a factor of 10 , inspired by the conclusions of the solar statistical study of the flare energy partition presented in Kretzschmar (2011).

Recently, Vida et al. (2019) performed a statistical analysis using archival stellar spectra and looked for line asymmetries. They found about 500 events with such asymmetries. As they note, most of those events showed enhanced Balmer line asymmetries, pointing to an association with flares. Vida et al. (2016) observed speeds of the order of $100-300 \mathrm{~km} \mathrm{~s}^{-1}$ and masses of the order of $10^{15}-10^{18} \mathrm{~g}$. The authors were then able to fit their results with a log-linear relation between event masses and speeds,

$$
\log M_{\text {blue }}=(12.67 \pm 0.17) v_{\text {blue }}^{0.050 \pm 0.003},
$$

with $M_{\text {blue }}$ being the mass of the blueshifted material and $v_{\text {blue }}$ its blueshifted speed. Equation (3) indicates that the masses and speeds of more "energetic" events increase simultaneously, with masses increasing much faster and extending to more than 3 orders of magnitude than event speeds, which only differ by a factor of a few. Individual event characteristic measurements were not reported in Vida et al. (2019), and not all of the events analyzed therein were associated with Balmer line enhancements. Thus, we cannot include them in the current analysis.

\subsection{Events Observed through X-Ray Absorption}

For the CME candidates observed through X-ray continuous absorption, we follow a similar approach as the one presented in Moschou et al. (2017). More specifically, we use the CME cone model, which is a geometric model often used for the analysis of CME events in the solar context (see, e.g., Howard et al. 1982; Fisher \& Munro 1984; Zhao et al. 2002; Xie et al. 2004) to estimate the CME characteristics. The CME cone model requires a density for the transient plasma, a characteristic length and timescale, to determine the CME cruising speed, an opening angle of the $\mathrm{CME}$ cone, and a thickness for the front of the conical shell. For the estimation of the CME mass, we use the column density increase captured in each observation. As the characteristic time, we choose the half-time of the column density decay, i.e., $\tau=t\left(N_{\mathrm{H}, \max } / 2\right)$, when possible. Then, based on the particular event, we define a lower (flaring/obscuring) length scale to be equal to the flaring loop size and an upper (dynamic) length scale for the CME to be equal to five times the stellar radius (see, e.g., Moschou et al. 2017). In terms of opening angles, we assume a semi-opening angle of $90^{\circ}$, since, as was argued in Aarnio et al. (2011), energetic events have wider opening angles and, as was shown in Moschou et al. (2017), there is a less than a factor of 2 difference between our calculations for $75^{\circ}$ and $90^{\circ}$. Finally, we define the conical shell's thickness as one-fifth of each length scale, i.e., $L_{\mathrm{obs}} / 5$ and $L_{\mathrm{dyn}} / 5$. All of the equations used for our analysis are detailed in Moschou et al. (2017).

\section{Stellar CME Candidates}

\subsection{Doppler-shift Method}

The CME candidates detected with the method of Dopplershifted emission, mainly in Balmer lines, are presented in Table 1. The observed temperatures indicate that it is chromospheric material that is moving away from the host star. Here we describe the main characteristics of each event currently available. For the evaluation of the possibility of a Doppler-based method, we are interested in the mass and outflow speed measured at a particular astrocentric height of the host star. We can then estimate the local star-specific escape speed at the height of the CME measurement and compare it with the observed CME speed in order to gain insight on how likely that particular event is to escape the gravitational attraction of the star (see, e.g., Guenther \& Emerson 1997; Bond et al. 2001; Fuhrmeister \& Schmitt 2004). The events are mentioned in chronological order of occurrence. It is important to note that Doppler-shift measurements only serve as lower limits in the observed outflow speeds, as they only measure the velocity component along the line of sight, thus suffering from large errors due to projection effects (for more, see Section 5). It is difficult to follow an escaping CME in chromospheric lines, as its mass will eventually get heated as it mixes with the hot $\geqslant 10^{6} \mathrm{~K}$ stellar corona. In Section 2.2 we discuss in detail how we analyze these events and convert their fluence to X-ray fluence. All analyzable events are also included in Figure 2, with the nonescaping ones as lower limits and different colors.

\subsubsection{AD Leo, 1984 March}

The best representative case for observing outflows through Doppler shifts, and one of the most promising CME candidates so far, was captured by Houdebine et al. (1990). The observed star was AD Leo, with $M=0.42 M_{\odot}$ and $R_{\star}=0.46 R_{\odot}$, which is a very active $\mathrm{M} 3.5 \mathrm{~V}$ (dMe) dwarf that produced a very powerful flare. The observation is unique in capturing line-ofsight plasma speeds as high as $5800 \mathrm{~km} \mathrm{~s}^{-1}$.

Houdebine et al. (1990) captured the mass outflow signature as a large blueshift in the Balmer lines $\mathrm{H}_{\gamma}$ and $\mathrm{H}_{\delta}$ using the 3.6 $\mathrm{m}$ telescope at the European Southern Observatory (ESO). The strong blueshift (outflow) was measured during the impulsive flare phase and lasted for a few minutes, while only a weak redwing enhancement was present indicating a simultaneous 
Table 1

Summary of All Events Potentially Linked to CMEs Observed through Doppler Shifts

\begin{tabular}{lccccccccc}
\hline $\begin{array}{l}\text { References } \\
(1)\end{array}$ & $\begin{array}{c}\text { Star } \\
(2)\end{array}$ & $\begin{array}{c}\text { Type } \\
(3)\end{array}$ & $\begin{array}{c}D(\mathrm{pc}) \\
(4)\end{array}$ & $\begin{array}{c}\text { Instrument } \\
(5)\end{array}$ & $\begin{array}{c}u_{\text {esc }} \\
(6)\end{array}$ & $\begin{array}{c}u_{\text {blue }} \\
(7)\end{array}$ & $\begin{array}{c}M(\mathrm{~g}) \\
(8)\end{array}$ & $\begin{array}{c}F(\mathrm{erg}) \\
(9)\end{array}$ & $\begin{array}{c}E_{k}(\mathrm{erg}) \\
(10)\end{array}$ \\
\hline 1 & AD Leo & M4Vae & 5 & ESO, IDS & 580 & $1500-5830$ & $>7.7 \times 10^{17}$ & $5 \times 10^{31}$ & $9 \times 10^{33 \mathrm{a}}-10^{35}$ \\
2 & AT Mic & M4.5Ve + M4.5Ve & 11 & SAAO & 500 & 600 & $10^{14}-10^{16}$ & $3 \times 10^{31}$ & $2-200 \times 10^{29}$ \\
3 & Cham J1149.8-7850 & wTTs & $140^{\mathrm{b}}$ & FLAIR II & 440 & 600 & $1.4-78 \times 10^{18}$ & $2 \times 10^{33}-10^{34}$ & $2.5-140 \times 10^{33}$ \\
4 & AU Mic & M1VeBa1 & 10 & $E U V E$ & 375 & 1400 & $10^{20}$ & $3 \times 10^{35}$ & $10^{36}$ \\
5 & V471 Tau & K2V+DA & 48 & GHRS & 550 & $>120$ & $>3 \times 10^{15}$ & $\cdots$ \\
6 & DENIS 1048-39 & M9 & $4.6^{\mathrm{d}}$ & DENIS & 550 & 100 & $3-30 \times 10^{16}$ & $9 \times 10^{29}$ & $1.5-15 \times 10^{29}$ \\
7 & AD Leo & M4Vae & 5 & FUSE & 580 & 84 & $4-500 \times 10^{30}$ & $2 \times 10^{31}$ & $1.5-150 \times 10^{31}$ \\
8 & V374 Peg & M3.5Ve & 9.1 & RCC & 580 & 675 & $10^{16}-10^{17}$ & $\sim 10^{33}$ & $2-20 \times 10^{31}$ \\
\hline
\end{tabular}

Notes. Column 1 indicates the original reference, column 2 the star observed, column 3 its spectral type, column 4 its distance in pc, column 5 the instrument, columns 6 and 7 the escape speed and blueshift speed in $\mathrm{km} \mathrm{s}^{-1}$, column 8 the mass of the event, column 9 the observed total radiated energy, and column 10 the kinetic energy of the event $E_{k}$. All distances are calculated with the SIMBAD database (Ochsenbein et al. 2000) parallaxes unless otherwise indicated. Spectral types are also given by SIMBAD.

${ }^{a} E_{k}$ estimated in Houdebine et al. (1990).

b Distance given in Guenther \& Emerson (1997).

${ }^{c}$ Spectral type classification as reported in Fuhrmeister \& Schmitt (2004).

${ }^{\mathrm{d}}$ Distance given in Fuhrmeister \& Schmitt (2004).

References. (1) Houdebine et al. (1990), (2) Gunn et al. (1994), (3) Guenther \& Emerson (1997), (4) Katsova et al. (1999), (5) Bond et al. (2001), (6) Fuhrmeister \& Schmitt (2004), (7) Leitzinger et al. (2011), and (8) Vida et al. (2016).

downflow. Before the flare onset, a faint absorption signature was measured in the $\mathrm{Ca}$ II $\mathrm{H}$ and $\mathrm{K}$ lines corresponding to $100 \mathrm{~km} \mathrm{~s}^{-1}$ with respect to the quiescent emission signal. The inferred opacity from the $\mathrm{H}_{\gamma} / \mathrm{H}_{\delta}$ line ratio measurements suggests that the plasma in the flaring region was forced to expand rapidly with its initial larger-than-the-mean-chromospheric opacity gradually decreasing. The fast-expanding plasma scenario is consistent with the large speeds observed.

Houdebine et al. (1990) emphasized that initially, the $\mathrm{H}_{\gamma} / \mathrm{H}_{\delta}$ decrements of the flare and flow were similar, which is evidence that the two plasma elements may come from the same atmospheric region. In the first few minutes after the flare onset, there was a strong deceleration, with the speed transitioning from 5830 to $3700 \mathrm{~km} \mathrm{~s}^{-1}$, and then it remained more or less constant. This could plausibly be the impulsive phase of a CME, during which the CME speed is much larger than the stellar wind speed, and it decelerates due to the drag force (Vršnak et al. 2004; Žic et al. 2015). A deceleration due to the interaction with the stellar wind is consistent with the small gravitational effect that Houdebine et al. (1990) estimated. The escape speed for AD Leo was calculated to be $u_{\mathrm{esc}} \sim 580 \mathrm{~km} \mathrm{~s}^{-1}$. A minimum speed component of about $\sim 1500 \mathrm{~km} \mathrm{~s}^{-1}$ was also measured during the first few minutes.

Houdebine et al. (1990) estimated a plasma density on the order of $n>10^{10} \mathrm{~cm}^{-3}$, which led to an estimated CME mass of $M=7.7 \times 10^{17} \mathrm{~g}$ and a kinetic energy of $E_{k}=5 \times 10^{34} \mathrm{erg}$, for a plasma with temperatures of $15,000-20,000$ K. From photometry, Houdebine et al. (1990) inferred a total radiated energy on the order of $F_{\text {broadband,tot }}=\left(3 \times 10^{28}-10^{35}\right)$ erg. For a flux varying from $f_{\mathrm{H}_{\gamma}}=6.23 \times 10^{-13}$ to $f_{\mathrm{H}_{\gamma}}=1.7 \times 10^{-13} \mathrm{erg} \mathrm{cm}^{-2} \mathrm{~s}^{-1}$ in 7 minutes for $\mathrm{AD}$ Leo, we get an $\mathrm{H}_{\gamma}$ fluence of $F_{\mathrm{H}_{\gamma}}=5 \times 10^{31} \mathrm{erg}$. Then, using Equation (1), we get an average X-ray fluence of $F_{\mathrm{X}} \approx 2 \times 10^{33} \mathrm{erg}$. The CME scenario explored therein resulted in an event with a mass and kinetic energy several times larger than any solar CME, which led the authors to name such an event a super-CME and conclude that in very active stars, there is the possibility of such transient events.

Leitzinger et al. (2011) used archival and published data from the Far-Ultraviolet Spectroscopic Explorer (FUSE) to study activity phenomena on the flare star AD Leo in the farUV. They used observational work from Christian et al. (2006), where two different flares were captured. A blue-wing enhancement in the O VI $1032 \AA$ line was produced by $\mathrm{AD}$ Leo after the flare event. The analysis of Leitzinger et al. (2011) favored the dynamic CME scenario to explain this emission feature. The authors were able to exclude other possibilities that could give rise to this feature, such as corotating gas clouds in a static scenario, as the enhancement was only captured in a single spectrum. A direct interstellar medium (ISM) column density observation by Wood et al. (2005) was used to correct for (ISM) absorption. The upflow speed in the AD Leo case was estimated to be $\sim 84 \mathrm{~km} \mathrm{~s}^{-1}$, similar to the velocity of chromospheric evaporation in the solar transition region, which was measured to be $\sim 100 \mathrm{~km} \mathrm{~s}^{-1}$ (Teriaca et al. 2003). Leitzinger et al. (2011) argued that in the case where projection effects are extreme, for example, when the CME propagates at an angle of $90^{\circ}$ from the line of sight, there will be no projected speed, and, for a measured speed of $\sim 100 \mathrm{~km} \mathrm{~s}^{-1}$, an actual CME speed of the order of $5730 \mathrm{~km} \mathrm{~s}^{-1}$ could be present. Finally, Leitzinger et al. (2011) excluded a prominence scenario by calculating a corotation radius smaller than the estimated possible cloud height, $R=27.9 R_{*} \approx 12 R_{\odot}$. The authors favored the scenario of a CME with extreme projection effects lowering its actual speed. Finally, if we assume a plasma density of $n_{\mathrm{H}}=10^{10}-10^{11} \mathrm{~cm}^{-3}$ and an emitting volume of $2.5 \times 10^{31}-3 \times 10^{33} \mathrm{~cm}^{-3}$, the estimated mass is $M \sim 4 \times 10^{17}-5 \times 10^{19} \mathrm{~g}$. Also, from Figure 1 in Leitzinger et al. (2011), for a duration of $200 \mathrm{~s}$, the AD Leo flare emitted energy of $\sim 2 \times 10^{31} \mathrm{erg}$ in the C III multiplet.

\subsubsection{AT Mic, 1992 May}

A large flare with total emitted energy in the wavelength range $3600-4200 \AA$ of $3 \times 10^{31} \mathrm{erg}$ was observed in an active 

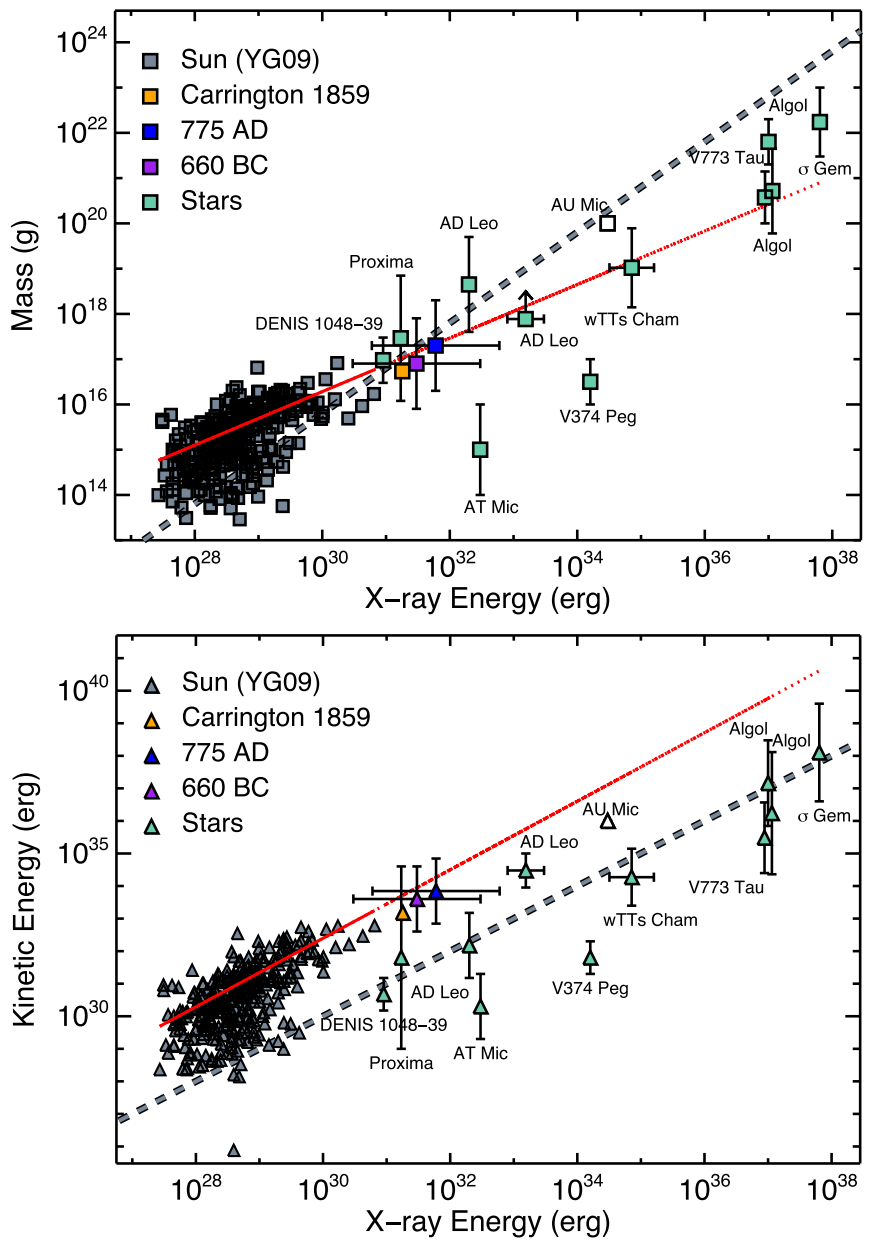

Figure 2. Derived CME masses (top) and kinetic energies (bottom) as a function of the associated flare total X-ray radiated energy in the GOES 1-8 band for solar events and the stellar events analyzed here and by Moschou et al. (2017). Solar events are from the compilation of Yashiro \& Gopalswamy (2009) and indicated by filled gray symbols. The Carrington event and the historic $775 \mathrm{AD}$ and $660 \mathrm{BC}$ events proposed by Melott \& Thomas (2012) and O'Hare et al. (2019), respectively, are also indicated (see text for details). Individual stellar events indicated by green filled symbols are labeled with the identity of the host star. The point corresponding to the AU Mic event is shown open to designate its more doubtful CME status (see text). The red lines represent the fit to the solar data by Drake et al. (2013). The gray dashed line in the upper panel represents a constant ratio of CME mass to flare X-ray energy. In the lower panel, the gray dashed line represents parity between flare X-ray and CME kinetic energies (see Drake et al. 2013 for further details).

M dwarf star, AT Mic (dM4.5e, $M_{*}=0.27 M_{\odot}$, $R_{*}=0.41 R_{\odot}$ ), by Gunn et al. (1994). Spectroscopic observations were performed using the RPCS spectrograph of the SAAO $1.9 \mathrm{~m}$ telescope in 1992 May. Both the flare and quiescent emission were clearly seen in the $\mathrm{H}_{\delta}$ and Ca II lines. A strong blue asymmetry, which is an indication of a bulk outflow, was captured in the Balmer emission lines. Gunn et al. (1994) noted that $200-400 \mathrm{~km} \mathrm{~s}^{-1}$ upflows in CaXIX and Fe XXV have been observed for solar events (Antonucci et al. 1982; Hara et al. 2011). A maximum line-of-sight speed of $\approx 600 \mathrm{~km} \mathrm{~s}^{-1}$, which decreased as the flare gradually decayed, was estimated. The escape speed at the surface of AT Mic was $500 \mathrm{~km} \mathrm{~s}^{-1}$. Chromospheric electron densities were taken as $n_{e} \sim 10^{12}-10^{14} \mathrm{~cm}^{-3}$, and the mass of the cool $T \sim 10^{4} \mathrm{~K}$ plasma was estimated to be $10^{15} \mathrm{~g}$, which then gave a kinetic energy of $E_{k} \sim 3 \times 10^{29} \mathrm{erg}$. Gunn et al. (1994) emphasized that the estimated plasma parameters have large uncertainties; however, they concluded that the mass flow kinetic energy was significantly less than the emitted flare energy.

\subsection{3. wTTs Cham, J1149.8-7850}

Guenther \& Emerson (1997) simultaneously observed 18 classical and 18 weak-line T Tauri stars (wTTs) using the FLAIR II spectrograph on the UK Schmidt telescope over $14 \mathrm{hr}$. The authors argued that wTTs generally show nonvariable emission over timescales of $>100 \mathrm{hr}$. However, two flares were captured from wTTs, both of which showed a fast rise phase and a longer decay lasting about $1 \mathrm{hr}$ in $\mathrm{H}_{\alpha}$. The wTTs J1149.8-7850 showed a flare with a 24 minute rise phase and a slower decline phase that lasted about $\sim 160$ minutes. A large blue asymmetry was measured with speeds reaching $-1000 \mathrm{~km} \mathrm{~s}^{-1}$ during the flare peak and a total energy reaching $\sim(2 \pm 0.7) \times 10^{33}$ erg in $\mathrm{H}_{\alpha}$. The estimated temperature of the flaring material was $20,000-30,000 \mathrm{~K}$.

The second flaring star was J1150.9-7411, whose flare had a 30 minute rise time with a decay observed over the following 2 hr. After this period, the emission had not yet reached quiescent levels, and Guenther \& Emerson (1997) estimated that the decay phase went on for another $2 \mathrm{hr}$. Thus, only a lower limit to the total energy release of $\geqslant(6 \pm 2) \times 10^{32} \mathrm{erg}$ was found. The plasma had a temperature of $<30,000 \mathrm{~K}$, and the blue and red wings remained unchanged during the event. The two flares from wTTs stars were 700 and 200 times more energetic than solar events that have not been observed to release more than $3 \times 10^{30}$ erg in $\mathrm{H}_{\alpha}$ (Somov 1992).

The J1149.8-7850 event had a pronounced blue asymmetry in its flare profile, with one component at the star rest speed and another at $-600 \mathrm{~km} \mathrm{~s}^{-1}$. This blueshift was interpreted as a CME by Guenther \& Emerson (1997), as the outflow was faster than the local escape speed, which for a wTTs is $v_{\mathrm{esc}}=440 \mathrm{~km} \mathrm{~s}^{-1}$ for $M_{\star}=M_{\odot}$ and $R_{\star}=2 R_{\odot}$. Guenther \& Emerson (1997) estimated the lower limits of the mass at $1.4-78 \times 10^{18} \mathrm{~g}, \quad$ an emitting volume of $6.2 \times 10^{31}-3.5 \times 10^{33} \mathrm{~cm}^{-3}$, and a kinetic energy of $2.5 \times 10^{33}-1.4 \times 10^{35} \mathrm{erg}$ using a density of $10^{10} \mathrm{~cm}^{-3}$. The total flux in the optical regime was inferred to be $\sim 10^{34} \mathrm{erg}$. Guenther \& Emerson (1997) estimated a magnetic field of 10-200 G, and they noted that the difference with respect to solar flares was the emitting volume and energy release, not the magnetic flux density.

\subsubsection{V471 Tau, 1994 October}

The Goddard High Resolution Spectrograph (GHRS) on the Hubble Space Telescope was used by Bond et al. (2001) to perform an UV spectroscopic observation of the precataclysmic binary V471 Tau, which consists of a white dwarf and a dK2 star and an orbital period of $12.51 \mathrm{hr}$ (Guinan \& Sion 1984). The $\mathrm{K}$ dwarf is tidally locked with the white dwarf and, consequently, rotates 50 times faster than the Sun and is thus much more active.

The white dwarf acts as a UV background in spectra of the system. Evidence for a transient was found in the form of absorption features in the white dwarf emission. Bond et al. (2001) argued that, since the V471 binary is a detached binary system in which neither star fills its Roche lobe, the CME evidence found must originate from processes similar to solar CMEs or, in other words, similar to an event originating from a 
single star. Wide Ly $\alpha$ absorption wings were captured in the spectra.

Bond et al. (2001) inferred a speed of $v>120 \mathrm{~km} \mathrm{~s}^{-1}$ for the absorber from the line-of-sight-measured speed of $\approx 20 \mathrm{~km} \mathrm{~s}^{-1}$ that was seen to cross the line of sight of the observation almost transversely. The duration of the obscuring mass flow was $1600 \mathrm{~s}$. Bond et al. (2001) inferred an extent for the CME of the order of $\geqslant 1.9 \times 10^{10} \mathrm{~cm}$, i.e., $L_{\mathrm{CME}} \sim 0.28 R_{\odot} \approx 0.29 R_{\star}$, and calculated possible launch paths for the CME. They found trajectories that leave the $\mathrm{K} 2$ dwarf at a specific orbital phase and pass in front of the white dwarf with a speed of $\sim 22 \mathrm{~km} \mathrm{~s}^{-1}$, triggering absorption features in the spectra and finally leaving the binary system. Other launch trajectories and speeds were also explored, and Bond et al. (2001) reported optimal velocities in the range $450-500 \mathrm{~km} \mathrm{~s}^{-1}$, consistent with solar observations (Yashiro \& Gopalswamy 2009). Bond et al. (2001) estimated the upper limit for the number density of the absorbing mass to be $n_{\mathrm{H}} \approx 6 \times 10^{11} \mathrm{~cm}^{-3}$ and the mass to be $M_{\mathrm{CME}}>3 \times 10^{15} \mathrm{~g}$.

\subsubsection{DENIS 1048-39, 2002 March}

The DENIS 1048-39 M9 dwarf was discovered by the DEep Near Infrared Survey (DENIS) at a distance of $4.6 \mathrm{pc}$. DENIS 1048-39 has an age of 1-2 Gyr, a radius of $0.1 R_{\odot}$, and a mass of $0.09 M_{\odot}$ and is at the lower mass limit of hydrogen-burning objects. However, as its $\mathrm{H}_{\alpha}$ variable emission shows, it still displays observable magnetic activity levels. Fuhrmeister \& Schmitt (2004) performed observations of DENIS 1048-39 using the Ultraviolet-Visual Echelle Spectrograph of the ESO Kueyen telescope. A blueshift of the order of $100 \mathrm{~km} \mathrm{~s}^{-1}$ was observed in the $\mathrm{H}_{\alpha}$ and $\mathrm{H}_{\beta}$ lines. A blueshift asymmetry was observed in both flare and background spectra that was strong in the $\mathrm{H}_{\alpha}$ line.

The $\mathrm{H}_{\alpha}$ emission corresponds to a temperature of approximately $T=10^{4} \mathrm{~K}$. The measured luminosity of $\mathrm{H}_{\alpha}$ was estimated at $1.6 \times 10^{26} \mathrm{erg} \mathrm{s}^{-1}$ for a $4.6 \mathrm{pc}$ distance, which gives a fractional luminosity of $\log \left(L_{\mathrm{H}_{\alpha}} / L_{\mathrm{bol}}\right)=-4.0$. The observed half-widths of the $\mathrm{H}_{\alpha}$ and $\mathrm{H}_{\beta}$ lines were always lower than $20 \mathrm{~km} \mathrm{~s}^{-1}$, which indicates that the emission comes from a finite region on the stellar surface. Since for both lines, there is evidence for the presence of two components, Fuhrmeister \& Schmitt (2004) concluded that the emission could originate from two active regions.

The escape velocity of the M9 dwarf is $v_{\mathrm{esc}} \sim 550 \mathrm{~km} \mathrm{~s}^{-1}$, while the observed projected velocity is $100 \mathrm{~km} \mathrm{~s}^{-1}$. However, the blueshift only provides a lower limit for the outflow. After 20 minutes of exposure, Fuhrmeister \& Schmitt (2004) estimated a $1.7 R_{\star}$ altitude for the mass, which is larger than the corotation distance, thus excluding a prominence scenario. Fuhrmeister \& Schmitt (2004) discussed the possibility that the measured projected velocity is a result of the integration through different velocities during a possible initial $\mathrm{CME}$ deceleration phase. For a Balmer line brightening lasting for $1.5 \mathrm{hr}$, we have a total radiated energy of $F_{\mathrm{H}_{\alpha}}=9 \times 10^{29} \mathrm{erg}$. Finally, if we assume a hydrogen plasma number density of $n_{\mathrm{H}}=10^{10}-10^{11} \mathrm{~cm}^{-3}$, the estimated CME mass is $M \sim 3 \times 10^{16}-3 \times 10^{17} \mathrm{~g}$ with a kinetic energy of $1.5 \times 10^{30}-1.5 \times 10^{31} \mathrm{erg}$.
3.1.6. V374 Peg, 2005

A 200 Myr old ultrafast rotating single M4 dwarf, V374 Peg has a rotation period of 0.44 days and is located 8.9 pc away. Vida et al. (2016) performed spectroscopic and photometric observations of V374 Peg using various observational facilities expanding over $16 \mathrm{yr}$. They concluded that V374 Peg has a magnetic field and a star-spot configuration that remain very stable over a time span of $16 \mathrm{yr}$. There was no indication of cyclic star-spot activity.

On HJD 2453603, Vida et al. (2016) observed three consecutive blue-wing enhancements in the Balmer lines, which suggests that they are related and resemble sympathetic solar flares. The $\mathrm{H}_{\alpha}$ line was observed to be constantly in intense emission. The three blue-wing enhancements occurred at a specific rotational phase, which indicates long-lived magnetic loop systems that trigger flares. Vida et al. (2016) argued that all three events came from the same active region nest. The long duration of the observation $(>10 \mathrm{hr})$ allowed for a better understanding of the intensity variability before and after the enhancements.

All three blue-wing asymmetries occurred during a single flare with flaring energy $\sim 10^{33} \mathrm{erg}$, with the third event being the strongest, corresponding to projected speeds of $675 \mathrm{~km} \mathrm{~s}^{-1}$. For its estimated mass of $M=0.3 M_{\odot}$ and radius of $R=0.34 R_{\odot}$, Vida et al. (2016) deduced an escape velocity for V374 Peg of $v_{\mathrm{esc}}=580 \mathrm{~km} \mathrm{~s}^{-1}$. They argued that the two first precursors $\left(350 \mathrm{~km} \mathrm{~s}^{-1}\right.$ each) could be failed eruptions, with the red-wing enhancement indicating material falling back after the initial upflow.

Failed eruptions are common phenomena in the Sun (e.g., Joshi et al. 2013; Zuccarello et al. 2017). A strong asymmetry was observed in a failed flux-rope eruption by Joshi et al. (2013), with a CME core getting formed. While the CME managed to lift off, it was then observed to fall back toward the Sun. The scientists suggested that overlying magnetic flux tubes covering part of the initial filament could be a reason for the ballistic behavior of the CME. In recent work, Zuccarello et al. (2017) studied the transition between eruptive flares (associated with a CME) and failed eruptions. They concluded that failed eruptions occur when the supporting field of the filament and the overlying field gradually reach a less antiparallel relative direction due to continuous photospheric shearing motions.

However, the last of the three V374 Peg events that were inferred by Vida et al. (2016) to have occurred almost along the line of sight had a projected (but close to true) speed of $675 \mathrm{~km} \mathrm{~s}^{-1}$, and this material escaped the gravitational attraction of the star. The authors estimated a CME mass of $M_{\mathrm{CME}} \sim 10^{16}-10^{17} \mathrm{~g}$ ( 1 order of magnitude accuracy, as the authors noted) following the Houdebine et al. (1990) analysis, assuming a temperature $T=2 \times 10^{4} \mathrm{~K}$ and a density in the range $10^{10}-10^{12} \mathrm{~cm}^{-3}$. Then, Vida et al. (2016) adopted an $\mathrm{X}$-ray luminosity of $\log L_{\mathrm{X}} \sim 28.4 \mathrm{erg} \mathrm{s}^{-1}$, and they inferred the occurrence of 15-60 CMEs day ${ }^{-1}$ with masses $M_{\mathrm{CME}}>10^{16} \mathrm{~g}$.

\subsection{X-Ray Absorption Method}

A valuable activity indicator, X-rays have a well-observed trend of increasing stellar activity with faster rotation to activity levels up to $10^{3}$ times higher than the current solar one. This trend, however, reaches a saturation regime wherein faster 
Table 2

A Summary of All Events Potentially Linked to CMEs Observed through X-Ray Absorption

\begin{tabular}{|c|c|c|c|c|c|c|c|c|c|}
\hline $\begin{array}{l}\text { References } \\
\text { (1) }\end{array}$ & $\begin{array}{l}\text { Star } \\
(2)\end{array}$ & $\begin{array}{l}\text { Type } \\
\text { (3) }\end{array}$ & $\begin{array}{l}D(\mathrm{pc}) \\
\quad(4)\end{array}$ & $\begin{array}{l}R_{\star}\left(R_{\odot}\right) \\
\quad(5)\end{array}$ & $\begin{array}{l}\text { Mission } \\
\text { (6) }\end{array}$ & $\begin{array}{c}L_{\text {peak }}^{\text {X-ray }}\left(\operatorname{erg~s}^{-1}\right) \\
(7)\end{array}$ & $\begin{array}{l}F(\text { erg) } \\
\quad(8)\end{array}$ & $\begin{array}{c}N_{\mathrm{H}, \max }\left(\mathrm{cm}^{-2}\right) \\
(9)\end{array}$ & $\begin{array}{l}T(\mathrm{~K}) \\
(10)\end{array}$ \\
\hline 9 & Algol B & K2IV & 28 & $3.5^{\mathrm{a}}$ & BepposAX & & $10^{37}$ & $3 \times 10^{21}$ & $1.4 \times 10^{8}$ \\
\hline 10 & Prox Cen & dM5.5Ve & 1.3 & $0.15^{\mathrm{b}}$ & Einstein & $2 \times 10^{28}$ & $3.5 \times 10^{31}$ & $10^{20}$ & $2.7 \times 10^{7}$ \\
\hline 12 & V773 Tau & $\mathrm{K} 3 \mathrm{Ve}$ & 128 & $4.17^{\mathrm{c}}$ & $A S C A$ & $10^{33}$ & $10^{37}$ & $4 \times 10^{22}$ & $10^{8}$ \\
\hline 13 & UX Arietis & $\mathrm{G} 5 \mathrm{IV}+\mathrm{K} 0 \mathrm{IV}$ & 51 & $1.6+5.6^{\mathrm{d}}$ & BepposAX & $10^{32}$ & $\geqslant 5 \times 10^{36}$ & $10^{20}$ & $10^{8}$ \\
\hline 14 & Pleiades & $\mathrm{K} 3 \mathrm{~V}+\mathrm{K} 3^{\mathrm{e}}$ & 120 & $0.7^{\mathrm{e}}$ & XMM-Newton & $\approx 10^{30}$ & $3 \times 10^{34}$ & $>10^{22}$ & $1.5 \times 10^{7}$ \\
\hline
\end{tabular}

Notes. Column 1 indicates the original reference, column 2 the star observed, column 3 its spectral type, column 4 its distance in pc, column 5 the stellar radius, column 6 the X-ray observatory, column 7 the peak X-ray luminosity, column 8 the observed X-ray fluence, column 9 the inferred column density, and column 10 the temperatures, which are based on plasma model fits performed in the original papers. All distances are calculated with the SIMBAD database (Ochsenbein et al. 2000) parallaxes unless otherwise indicated. Spectral types are also given by SIMBAD.

a Algol radius as reported in Richards (1993).

${ }^{\mathrm{b}}$ Proxima radius as reported in Kervella et al. (2017).

${ }^{c}$ V773 Tau radius as reported in Tsuboi et al. (1998).

${ }^{\mathrm{d}}$ UX Arietis radius as reported in Hummel et al. (2017).

e Pleiades spectral type and radius as reported in Briggs \& Pye (2003).

$\mathrm{f} \sigma$ Gem radius as reported in Roettenbacher et al. (2015).

References. Continuing enumeration from Table 1. (9) Favata \& Schmitt (1999), (10) Haisch et al. (1983), (11) Ottmann \& Schmitt (1996), (12) Tsuboi et al. (1998), (13) Franciosini et al. (2001), (14) Briggs \& Pye (2003), and (15) Pandey \& Singh (2012).

rotators cannot exceed fractional X-ray luminosities of the order of $L_{\mathrm{X}} / L_{\mathrm{bol}}=10^{-3}$ (Feigelson et al. 2004; Schmitt \& Liefke 2004; Telleschi et al. 2007; Wright et al. 2011, 2018) for both partially convective and $\mathrm{M}$ dwarf stars. While stellar coronae are unresolvable with current $\mathrm{X}$-ray telescopes, flaring loop characteristics can be inferred by analyzing the flare decay profiles (Reale et al. 1997; Reale 2007).

Several X-ray events with continuous enhanced absorption have been observed in different late-type active flaring stars. The largest events have been observed on short-period binary stars, such as Algol-like and RS CVn-type systems, as well as on young fast rotators (e.g., Graffagnino et al. 1995; Grosso et al. 1997; Tsuboi et al. 2000). The working hypothesis for these events is that a mass of plasma is obscuring the flaring region and, while escaping the gravitational potential of the star, it also expands, causing a characteristic decay of the absorbing column density.

In an earlier study, we analyzed the most prominent representative of such an event (Moschou et al. 2017) by assuming that a CME was ejected. Here we use the CME cone geometric model, similar to the analysis presented in Moschou et al. (2017) for the Algol CME, for all of the X-ray absorption events observed so far (see Table 2).

\subsubsection{Algol Events}

1992 August event. Ottmann \& Schmitt (1996) observed Algol for 2.4 binary orbits with ROSAT/PSPC in the second half of 1992 August. A giant X-ray flare took place in the middle of the observation and lasted for half a binary orbit, i.e., about 1.5 days. To establish a baseline emission level and isolate the flare signal, Ottmann \& Schmitt (1996) adopted the quiescent light curve observed by Ottmann (1994). The beginning of the flare was considered to be $t_{0}=293,000 \mathrm{~s}$ into the observation. The deduced flare rise phase lasted for $24,000 \mathrm{~s}$, while the decay extended up to $100,000 \mathrm{~s}$. The authors estimated an $e$-folding time from the light curve of $\tau=30,400$ s, i.e., more than $8 \mathrm{hr}$.
The Raymond \& Smith (1977) one-temperature thermal plasma model was applied to the data, indicating that the temperature and density peaked during the flare rise phase and reached $T \approx 10^{8} \mathrm{~K}$ and $n_{e} \approx 5 \times 10^{11} \mathrm{~cm}^{-3}$, respectively. The observations of Ottmann \& Schmitt (1996) are illustrated in the middle panel of the middle row of Figure 1 with errors at $90 \%$ confidence levels.

Ottmann \& Schmitt (1996) discussed a potential column density increase of a factor of 2 during the early decay phase, reaching $N_{\mathrm{H}} \approx 2 \times 10^{19} \mathrm{~cm}^{-2}$. Similar to Haisch et al. (1983), the authors characterized the giant Algol flare as a two-ribbon one. The flare had a thermal energy of $7 \times 10^{36} \mathrm{erg}$, a peak luminosity of $L_{\mathrm{X}}=2 \times 10^{32} \mathrm{erg} \mathrm{s}^{-1}$, and a flaring volume of $V=1.3 \times 10^{34} \mathrm{~cm}^{3}$. A flaring loop length of $5 \times 10^{11} \mathrm{~cm}^{-3}$, corresponding to an altitude of $0.65 R_{B}$, was estimated by applying a quasi-static cooling method.

1997 August event. An X-ray stellar flare that occurred on Algol on 1997 August 30 ranks among the most energetic stellar flares ever observed. The proximal $(28.5 \mathrm{pc})$ Algol prototypical system is a binary star with an early-type primary, which is a $\mathrm{B} 8 \mathrm{~V}\left(R_{A}=2.9 R_{\odot}\right)$ star, and a secondary K2IV $\left(R_{B}=3.5 R_{\odot}\right)$ star. The binary system is tidally locked and has a period of 2.7 days.

The flare observation was made by BeppoSAX/LECS, MECS, and PDS, and the event was analyzed by Favata \& Schmitt (1999), who found the total X-ray fluence to be approximately $1 \times 10^{37}$ erg in the 1-8 $\AA$ GOES band. Favata \& Schmitt (1999) also measured a quiescent volume emission measure of $\mathrm{EM}=n_{e}^{2} V=3 \times 10^{53} \mathrm{~cm}^{-3}$. Shortly after the Algol superflare peak, the column density increased significantly (by $\sim 2$ orders of magnitude) from the $\approx 10^{20} \mathrm{~cm}^{-2}$ base value and then gradually decayed with an $e$-folding time of $\tau=5.6 \mathrm{ks} \approx 1.5 \mathrm{hr}$ (Moschou et al. 2017).

The scenario of a CME being responsible for the X-ray continuous absorption and the column density variability was explored by Moschou et al. (2017). Those authors analyzed the column density profile and showed that it followed a $\propto t^{-2}$ law, which is consistent with a quasi-constant CME propagation and 
expansion. Using physical arguments, Moschou et al. (2017) defined two length scales for the CME evolution: a dynamic one based on the length scale established by solar studies (Žic et al. 2015), where the CME pressure balances the solar wind one, and a minimum length scale such that the CME just obscures the flaring region initially. Then, using (a) the derived temporal relation for the column density $\left(N_{\mathrm{H}} \propto t^{-2}\right)$, which in that case was consistent with a CME expanding self-similarly; (b) the geometric CME cone model often used in solar cases (Howard et al. 1982; Fisher \& Munro 1984; Zhao et al. 2002; Xie et al. 2004); and (c) geometric arguments about the flaring site, Moschou et al. (2017) obtained lower and upper limits for the $\mathrm{CME}$ mass and kinetic energy in the ranges $2 \times 10^{21}-2 \times 10^{22} \mathrm{~g}$ and $7 \times 10^{35}-3 \times 10^{38} \mathrm{erg}$, respectively.

\subsubsection{Proxima Centauri, 1980 August}

A $5 \mathrm{hr}$ observation of the dM5e star Proxima Centauri $\left(R_{*}=0.15 R_{\odot}\right)$ was performed by Haisch et al. (1983) simultaneously using observations from Einstein/IPC and the International Ultraviolet Explorer (IUE) in 1980 August. The authors reported indirect evidence of a two-ribbon flare, which is considered an indication of a prominence eruption in solar physics. The quiescent coronal luminosity was measured to be $L_{\text {cor }} \sim 5 \times 10^{26} \mathrm{erg} \mathrm{s}^{-1}$ and was subtracted from the X-ray flare light curve. Prox Cen has an X-ray-to-bolometric luminosity ratio 100 times larger than the Sun, reaching $L_{\text {cor }} / L_{\text {bol }} \sim 8 \times 10^{-5}-3 \times 10^{-4}$. The coronal temperature was estimated to be $T \sim 4 \times 10^{6} \mathrm{~K}$. For the flare, a peak was measured at $L_{x} \approx 2 \times 10^{28} \mathrm{erg} \mathrm{s}^{-1}$ (consistent with an X20 solar flare according to the $G O E S^{6}$ scale) and a maximum temperature of $\sim 27 \times 10^{6} \mathrm{~K}$. The observation lasted for the entirety of the flare, capturing both the pre- and post-flare luminosity levels. A best-fit $\chi^{2}$ thermal plasma model (see, e.g., Raymond \& Smith 1977) was used to calculate $T$ and $N_{\mathrm{H}}$ at $90 \%$ joint confidence levels, including all sources of uncertainty apart from the Einstein instrument calibration errors.

The estimated column density, $N_{\mathrm{H}}$, with the errors reported in Haisch et al. (1983), is illustrated in the left panel of the middle row of Figure 1. The column density increased immediately after the flare luminosity peak, reaching a value of $N_{\mathrm{H}} \approx 10^{20} \mathrm{~cm}^{-2}$, and then returned to quiescent pre-flare values of the order of $10^{18}-10^{19} \mathrm{~cm}^{-2}$. The authors reported an $e$-folding time $(1 / e)$ for the temperature and luminosity of the order of $\tau \approx 20$ minutes.

Using solar coronal loop models, Haisch et al. (1983) were able to estimate an X-ray emission measure of $\mathrm{EM} \approx 8.3 \times 10^{48} \mathrm{~cm}^{-3}$ for the coronal temperature, an upper limit for the flaring loop length $L \leqslant 2.4 \times 10^{9} \mathrm{~cm}$, and a lower limit for the coronal density $n \geqslant 7.5 \times 10^{9} \mathrm{~cm}^{-3}$. By comparing the Prox Cen flare to solar two-ribbon flares, Haisch et al. (1983) were able to estimate the total flaring energy at $E_{\mathrm{tot}}=3.5 \times 10^{31} \mathrm{erg}$. The authors discussed the possibility of cool, dense prominence material being the source of the sharp $N_{\mathrm{H}}$ increase to $10^{20} \mathrm{~cm}^{-2}$ shortly after the flare peak. This interpretation was consistent with solar two-ribbon flares that are often associated with transients and contain enough mass to cause the observed column density increase.

\footnotetext{
6 Solar flares are classified according to their energies into A-, B-, C-, M-, and $\mathrm{X}$-class events, ranging from the weakest to the most energetic ones. The flare classification in the GOES scale is based on the flare peak emission in the soft X-ray band of $1-8 \AA$.
}

Finally, using the simultaneous IUE UV measurements, Haisch et al. (1983) concluded that the radiative energy loss in the observed Prox Cen flare was more important in the corona than in the chromosphere or transition region, consistent with a dominant radiative cooling (rather than conduction cooling for the solar case) and maybe cooling due to expansion.

\subsubsection{V773 Tau, 1995 February}

In 1995 February, Tsuboi et al. (1998) obtained a $40 \mathrm{ks}$ $(\approx 11 \mathrm{hr})$ observation of the wTTs V773 Tau using ASCA/SIS, GIS. A spectroscopic binary, V773 Tau consists of K2V and K5V stars (Welty 1995). A strong flare was observed in which the X-ray emission increased by a factor of 20. The flare had a sharp rise phase and a slower exponential decay phase with an $e$-folding time of $\tau=8.2 \mathrm{ks} \approx 2.3 \mathrm{hr}$. More specifically, Tsuboi et al. (1998) reported a large lower limit for the peak flare luminosity of the order $L_{\mathrm{X}} \approx 10^{33} \mathrm{erg} \mathrm{s}^{-1}$ and a total energy reaching $E_{\mathrm{tot}} \approx 10^{37} \mathrm{erg}$, which were calculated after subtracting the quiescent X-ray spectrum obtained from their pre-flare measurements.

Tsuboi et al. (1998) noted that typical X-ray flares from TTs have luminosities $L_{\mathrm{X}} \geqslant 10^{31} \mathrm{erg} \mathrm{s}^{-1}$, i.e., 4 orders of magnitude more powerful than typical solar events (e.g., Montmerle et al. 1983; Preibisch et al. 1995; Kamata et al. 1997). Like solar flares, this rapid energy release is most likely due to magnetic reconnection. Strong magnetic activity in TTs is observed not only in X-rays but also in radio wavelengths that reach luminosities of the order $L_{R}=10^{18} \mathrm{erg} \mathrm{s}^{-1} \mathrm{~Hz}^{-1}$, often accompanied by circular polarization during flare and quiescent times (Phillips et al. 1991; White et al. 1992; Skinner 1993). Circular polarization offers direct evidence for the presence of magnetic fields.

After subtracting the pre-flare spectrum and using thermal plasma models, Tsuboi et al. (1998) were able to obtain a best fit and estimate the temperature and column density at each time bin. We illustrate their results and corresponding errors in the right panel of the middle row of Figure 1. The flaring material reached a maximum temperature of $T=10^{8} \mathrm{~K}$, a maximum column density of $N_{\mathrm{H}}=4 \times 10^{22} \mathrm{~cm}^{-2}$, and a volume emission measure of the order of $\mathrm{EM} \approx 10^{55} \mathrm{~cm}^{-3}$. The column density was significantly larger during the flare than in pre- or post-flare measurements. An agreement of broadband X-ray fluxes between Tsuboi et al. (1998) and Skinner (1993) indicates that V773 Tau has a constant X-ray emission level with $L_{\mathrm{X}} / L_{\mathrm{bol}}=10^{-3}$. Tsuboi et al. (1998) noted that the extreme energy release associated with the flare could cause expansion or evaporation of underlying material. Then, a quasi-static cooling loop model gave an electron density of $n_{e}=3 \times 10^{11} \mathrm{~cm}^{-3}$, a derived emitting volume of $V=6 \times 10^{32} \mathrm{~cm}^{3}$, and a loop size of $L=4 \times 10^{11} \mathrm{~cm}$, which is larger than the stellar radius, $R_{\star}=2.9 \times 10^{11} \mathrm{~cm}$, but still 10 times smaller than the semimajor axis of the system.

\subsubsection{UX Arietis}

Consisting of a G5IV and K0IV star, UX Ari is one of the most active RS CVn systems known. Observations in two sequential years, 1997 and 1998 August, of UX Ari with BeppoSAX and all three LECS, MECS, and PDS detectors were presented by Franciosini et al. (2001). An hour-long flare was observed during the first observation, but only a quiescent signal was picked up during the second one. Only the final $2 \mathrm{hr}$ 
of the flare rise phase were captured, as the flare appeared to have started before the observation began. The flare decay phase was longer, with an $e$-folding time $\tau \approx 13 \mathrm{hr} \approx 47 \mathrm{ks}$. Franciosini et al. (2001) noted that a contribution from rotational modulation cannot be ruled out, because the observations cover only slightly less than half an orbital period (0.4).

The spectra were fitted with two-temperature thermal plasma models using MEKAL (Mewe et al. 1995) and accounting for interstellar absorption. The best fit gave a column density of $N_{\mathrm{H}}=2.6 \times 10^{19} \mathrm{~cm}^{-2}$ for the quiescent (second) observation of 1998 August and $N_{\mathrm{H}} \approx \times 10^{20} \mathrm{~cm}^{-2}$ for the entire flare duration during 1997 August. In Figure 1, we illustrate in the left panel of the bottom row the column density profile from the best fit of Franciosini et al. (2001) and the errors reported therein. Franciosini et al. (2001) used the updated response matrix released in 2000 January for the LECS instrument, which solved the problem of systematically overestimating hydrogen column densities when observing stellar coronae with BeppoSAX. The old matrix gave a quiescent column density of $N_{\mathrm{H}}=8 \times 10^{19} \mathrm{~cm}^{-2}$, i.e., three times larger than the updated matrix, similar to what was found for all previous BeppoSAX observations, including Favata \& Schmitt (1999). However, the flare column density-even though it is 1.4 times lower than the one obtained with the old matrices - is still pretty high and, more specifically, about five times larger than the quiescent value.

The hard X-ray emission is fully attributed to the flarerelated hot plasma. The peak X-ray luminosity was $L_{\mathrm{X}} \approx 10^{32} \mathrm{erg} \mathrm{s}^{-1}$, reaching a value of $1.4 \times 10^{31} \mathrm{erg} \mathrm{s}^{-1}$ at the end of the flare. The peak emission measure was $\mathrm{EM} \sim 8 \times 10^{54} \mathrm{~cm}^{-3}$, and the peak temperature was $T=100 \mathrm{MK}$. The total X-ray energy release during the flare was $E_{\mathrm{X}} \geqslant 5 \times 10^{36} \mathrm{erg}$.

A two-ribbon flare model (Poletto et al. 1988) adopted from the solar original (Kopp \& Poletto 1984) was used to analyze the data, in which the magnetic energy released depends on the flaring region and the magnetic field strength. Constraining the surface magnetic field strength to be of $\mathrm{kG}$ order, according to other observations in RS CVn-type binaries, Franciosini et al. (2001) discussed that the flare took place in a $33^{\circ}-53^{\circ}$ region corresponding to an $\sim 0.6-0.9 R_{\star}$ width. The flaring loop halflength was estimated at $\sim 0.1-0.2 R_{\star}$ during the flare peak and $\sim 0.5-0.8 R_{\star}$ at the end of the flare. These results were in agreement with the ASCA flare analyzed by Güdel et al. (1999). For these estimations, the authors assumed an evaporation velocity of $500 \mathrm{~km} \mathrm{~s}^{-1}$. The X-ray emission amounted to only a small $0.1 \%-0.3 \%$ fraction of the total magnetic energy release. Finally, Franciosini et al. (2001) theorized that a CME and the resulting absorbing material along the line of sight could explain the column density increase during the flare by a factor of 5 with respect to the quiescent observation.

\subsubsection{LQ Hyades, 1992 November}

Covino et al. (2001) used ROSAT/PSPC and ASCA/SIS,GIS to observe GI 355, an active young star in LQ Hya in X-rays. The ROSAT observations were divided into eight parts lasting for $1500-2000 \mathrm{~s}$ each. ROSAT observed a large flare with a peak X-ray flux of $10^{31} \mathrm{erg} \mathrm{s}^{-1}$. The decay phase of the flare had an $e$-folding time $\tau \sim 10.1 \mathrm{ks}$ (Covino et al. 2001). Roughly 6 months after the ROSAT observations, ASCA observed GI 355 for about 20,000 s. For each ROSAT pointing, one- and two-temperature models with free metallicity $Z$ and column density $N_{\mathrm{H}}$ were used to fit the data. Covino et al. (2001) noted that they were not able to get a satisfactory spectral fit during the main part of the flare for ROSAT. Fits with one-, two-, and three-temperature models, fixed column density $N_{\mathrm{H}}=4 \times 10^{19} \mathrm{~cm}^{-2}$, and free metallicity were performed for ASCA data. The column density remained constant during the flare without any increase from flare onset to flare decay. The total emitted X-ray energy was $F_{\mathrm{X}}=9 \times 10^{34} \mathrm{erg}$. Covino et al. (2001) used the approximate density for the interstellar material found in Paresce (1984), $n_{\mathrm{H}}=0.07 \mathrm{~cm}^{-3}$, for obtaining an estimate of the column density of a star at $18 \mathrm{pc}$ (like GI 355) and got a column density of $N_{\mathrm{H}} \sim 4 \times 10^{18} \mathrm{~cm}^{-2}$, i.e., 1 order of magnitude lower than the fitted value. However, Covino et al. (2001) noted that they found no significant variation in the column density temporal profile to indicate the presence of a CME, only an increased value with respect to the value from the Paresce (1984) paper. We cannot analyze this event as a CME based on this analysis, as even if the column density is increased, there is no indication of a mass outflow. From solar physics, we know that CMEs do not always erupt during the flare onset at the flare site; however, an outgoing flow would present some decay with time if it were indeed captured in the flare spectra. However, the presence of prominence material cannot be excluded.

\subsubsection{Pleiades, H II 1100, 2000 September}

The Pleiades is a nearby young cluster with an age of $\approx 100 \mathrm{Myr}$ consisting of stars ranging from $\mathrm{B}$ to $\mathrm{M}$ types. These characteristics make it a useful target for studying X-rayproducing processes in coeval stars with different masses. Indeed, several X-ray telescopes have targeted the Pleiades, including Einstein, ROSAT, and Chandra (Micela et al. 1990; Stelzer \& Neuhäuser 2001; Daniel et al. 2002).

Of more interest for our study, the Pleiades was also observed in a $40 \mathrm{ks} \mathrm{X}$-ray survey with the XMM-Newton/ European Photon Imaging Camera (EPIC) by Briggs \& Pye (2003). During the observation, the H II 1100 binary star (Briggs \& Pye 2003 used H II number designations from Hertzsprung 1947, Table 2), consisting of twin K3 stars, produced a flare with decay time $\tau \sim 3 \mathrm{ks}$. The fractional quasi-steady X-ray luminosity of H II 1100 was measured to be $L_{\mathrm{X}} / L_{\mathrm{bol}}=-3.48$. The derived plasma temperature was in the range $14-17 \mathrm{MK}$ with an average of $15.4 \mathrm{MK}$, while the emission measure and X-ray flux at the flare peak were estimated to be $\mathrm{EM}=1.4 \times 10^{53} \mathrm{~cm}^{-3}$ and $L_{\mathrm{X}} \approx 10^{30} \mathrm{erg} \mathrm{s}^{-1}$, respectively. The flaring loop size was then inferred to be $1.2 \times 10^{10} \mathrm{~cm} \sim 0.25 R_{\star}$ using a hydrodynamic flare model scaling technique similar to Reale et al. (1997). A mean electron density in the flaring loop at the flare peak was estimated at $n_{e} \sim 5 \times 10^{11} \mathrm{~cm}^{-3}$, while the required magnetic field strength for confinement was $B \sim 300 \mathrm{G}$.

A sharp dip in the H II 1100 light curve was extensively discussed in Briggs \& Pye (2003), who examined various explanations, including an eclipse from an orbiting Jupitersized planet, obscuration of the flaring site by a CME, and the coincidence of two flares, with the first having a faster decay than rise. Briggs \& Pye (2003) did not favor any of these over the others. They noted that an eclipse by a Jupiter-like body has a low probability of occurrence. For a CME explanation, a column density of the order of $N_{\mathrm{H}}>10^{22} \mathrm{~cm}^{-2}$, which is 2 orders of magnitude higher than a typical solar prominence 
value, and velocities of $\sim 150-500 \mathrm{~km} \mathrm{~s}^{-1}$ are required. Figure 1 (middle panel, bottom row) shows the nominal column density for the Pleiades and the required one for a $\mathrm{CME}$ obscuration event. Briggs \& Pye (2003) estimated that such a CME would have a kinetic energy of the order of $E_{k} \sim 6 \times 10^{33} \mathrm{erg}$, which is comparable to the total flaring energy observed. Finally, assuming a flare duration of about $30 \mathrm{ks}$ and a constant flux of $L_{\mathrm{X}} \approx 10^{30} \mathrm{erg} \mathrm{s}^{-1}$, the total flaring energy becomes $E_{\mathrm{tot}}=3 \times 10^{34} \mathrm{erg}$.

\subsection{7. o Gem, 2001 April}

Seven flares from five binary systems of the RS CVn type were observed by Pandey \& Singh (2012) using XMM-Newton and EPIC. The strongest flare was observed in the RS CVn $\sigma$ Gem, which has an active K1III-type primary and an unobserved late-type main-sequence secondary. It was observed for $56 \mathrm{ks}$. During these measurements, only the flaring without the quiescent states was observed for $\sigma \mathrm{Gem}$. A quiescent state was then estimated using the Nordon et al. (2006) observation of $\sigma$ Gem with Chandra. Pandey \& Singh (2012) thus adopted a value of $25 \%$ lower flux with respect to the measured flare peak for the quiescent emission of $\sigma \mathrm{Gem}$. The X-ray peak luminosity for the $\sigma$ Gem flare is $L_{\mathrm{X}} \approx 10^{32} \mathrm{erg} \mathrm{s}^{-1}$, with a total emitted energy of the order of $F_{\mathrm{X}}=4.24 \times 10^{37} \mathrm{erg}$ and an $e$-folding flare decay time of $\tau \approx 32.5 \mathrm{ks}$. For obtaining the best fit in the $\sigma \mathrm{Gem}$ data, all one-, two-, and three-temperature collisional plasma models from Astrophysical Plasma Emission Code (APEC) were used (Smith et al. 2001). However, one- and two-temperature models using solar photospheric abundances could not fit the data well; thus, a best-fitting three-temperature model was used for $\sigma \mathrm{Gem}$. The derived column density was found to increase by a factor of $\sim 2$ during the flare rise phase and decrease during the flare decay phase. The maximum column density reached $N_{\mathrm{H}}=2.7 \times 10^{20} \mathrm{~cm}^{-2}$, i.e., twice as high as the quiescent value. Figure 1 (right panel, bottom row) shows the column density profile estimated in Pandey \& Singh (2012). The temperature and metallicity appear to peak at the flare onset phase. The emission measure reached a value of $\mathrm{EM}=3.77 \times 10^{54} \mathrm{~cm}^{-3}$, i.e., increased by a factor of $\sim 1.8$. A time-dependent hydrodynamic model (Reale et al. 1997, 2004; Reale 2007) was employed, similar to Pandey \& Singh (2008), to estimate the characteristics of a single flaring loop. The peak temperature for the $\sigma$ Gem flare was $177 \pm 6$ MK. The hydrodynamic flaring loop length was estimated to be $<6.43 \times 10^{11} \mathrm{~cm}$, while the rise- and decay-based estimations gave $>2.55 \times 10^{11}$ and $5.18 \times 10^{11} \mathrm{~cm}$, respectively. Assuming a fully ionized hydrogen plasma, an electron density of the order of $10^{10-11} \mathrm{~cm}^{-3}$ was found.

\subsection{BC Event}

The 775 AD event, shown in Figure 2 and further discussed in Moschou et al. (2017), is not the only case where significant enhancements of proton fluxes were inferred by studying radionuclides in ice cores. Recently, O'Hare et al. (2019) estimated the proton fluence for an event dated to $660 \mathrm{BC}$. The $660 \mathrm{BC}$ solar proton event was found to be 1 order of magnitude stronger than any solar event recorded during the instrumental period and of the same order of magnitude as the 775 AD event. O'Hare et al. (2019) estimated proton fluences of $\quad F_{30}=2.1 \times 10^{10}, \quad F_{100}=6.3 \times 10^{9}, \quad$ and
$F_{360}=1.6 \times 10^{9}$ protons $\mathrm{cm}^{-2}$ for protons with more than 30,100 , and $360 \mathrm{MeV}$ fluences, respectively.

Following a similar method as that presented in Cliver et al. (2014) and using available scalings from solar data, we provide the best guess for the characteristics of an associated CME and flare. First, using the broad scatter relation derived by Cliver \& Dietrich (2013), we find a best guess for the soft X-ray GOES $1-8 \AA$ equivalent fluence to the $F_{30}$ proton flux of the $660 \mathrm{BC}$ event of the order of $\sim 20 \mathrm{~J} \mathrm{~m}^{-2}$. The emitted soft X-ray energy is then $3 \times 10^{31} \mathrm{erg}$, which corresponds to a flare bolometric energy of $1.25 \times 10^{33} \mathrm{erg}$, according to Figure 3 in Cliver \& Dietrich (2013). Then, using the scatter relation of CME properties as a function of the associated flare X-ray fluence derived by Yashiro \& Gopalswamy (2009), we infer an associated CME mass of $8 \times 10^{16} \mathrm{~g}$ and kinetic energy $4 \times 10^{33} \mathrm{erg}$.

Here we have assumed a semi-opening angle of $90^{\circ}$ for the CME based on solar statistical studies for energetic X-class flares (Yashiro \& Gopalswamy 2009; Aarnio et al. 2011), as explained in Section 2 and thoroughly discussed in Moschou et al. (2017). This is significantly larger than the $24^{\circ}$ opening angle assumed by Melott \& Thomas (2012) for the 775 AD event. We take this opportunity to update the CME and flare parameters for the $775 \mathrm{AD}$ event to account for the larger, more likely opening angle. The resulting characteristics are $E_{k} \approx 7 \times 10^{33}$ erg for the CME kinetic energy, $2 \times 10^{17} \mathrm{~g}$ for the mass, $2.2 \times 10^{33}$ for the flare bolometric energy, and $6 \times 10^{31}$ erg for the flare energy emitted in soft X-rays.

We note that for these analyses, we rely on multiple scaling relations based on data with significant scatter (Yashiro \& Gopalswamy 2009; Cliver \& Dietrich 2013). As a result, different papers in the literature have inferred different kinetic energies for the $775 \mathrm{AD}$ event, from $2 \times 10^{33} \mathrm{erg}$ in Melott \& Thomas (2012), to $2 \times 10^{35} \mathrm{erg}$ in Miyake et al. (2012), to an intermediate value of $3 \times 10^{34} \mathrm{erg}$, as computed in Cliver et al. (2014). For this reason, in Figure 2, we have added error bars of a factor of 10 reflecting the scatter in Figure 15 of Cliver \& Dietrich (2013), which we used to infer the soft X-ray flaring energy. Using a factor of 10 for the X-ray flaring energy then translates into a similar factor of $\sim 10$ uncertainty for the inferred kinetic energy of the CME (see Figure 3 and discussion in Cliver \& Dietrich 2013). For the CME mass, we also use an error of a factor of 10 based on the spread of the solar data in Yashiro \& Gopalswamy (2009).

\subsection{AU Mic, 1992 July}

Cully et al. (1994) observed an EUV superflare with energy $3 \times 10^{35} \mathrm{erg}$ on AU Mic using the Extreme-Ultraviolet Explorer (EUVE) in 1992 July. Even though no direct evidence of a Doppler shift was found, a rapidly expanding CME was considered to explain the flare decay with a mass of $\sim 10^{20} \mathrm{~g}$ and a kinetic CME energy of $10^{36} \mathrm{erg}$, i.e., 4-5 orders of magnitude higher than solar events. Since $M_{*}=0.31 M_{\odot}$ and $R_{*}=0.84 R_{\odot}$, AU Mic has an escape speed of $375 \mathrm{~km} \mathrm{~s}^{-1}$. The mass and kinetic energy estimated therein correspond to a CME speed of $1400 \mathrm{~km} \mathrm{~s}^{-1}$, which is larger than the AU Mic local escape speed. Later, Katsova et al. (1999) argued that the CME explanation scenario is improbable given the lack of Doppler shifts or line broadening. Evidence pointed toward high plasma density, possibly reaching $10^{12} \mathrm{~cm}^{-3}$ during the flare decay rather than low densities expected of an expanding 
Table 3

Analyzable Stellar CME Candidates Using Doppler-shift (Top Half) and X-Ray (Bottom Half) Methods

\begin{tabular}{|c|c|c|c|c|c|c|c|}
\hline $\begin{array}{l}\text { Blueshift } \\
\text { (1) }\end{array}$ & $\begin{array}{l}\text { Star } \\
(2)\end{array}$ & $\begin{array}{c}u_{\mathrm{CME}}\left(\mathrm{km} \mathrm{s}^{-1}\right) \\
\text { (3) }\end{array}$ & $M_{\mathrm{CME}}(\mathrm{g})$ & $E_{k, \mathrm{CME}}(\mathrm{erg})$ & $\begin{array}{l}\text { Emission } \\
\text { (6) }\end{array}$ & $\begin{array}{l}F(\text { erg) } \\
\quad(7)\end{array}$ & $\begin{array}{c}F_{\mathrm{X}}(\mathrm{erg}) \\
(8)\end{array}$ \\
\hline$\overline{1}$ & AD Leo & $1500-5830$ & $>7.7 \times 10^{17}$ & $9 \times 10^{33}-10^{35}$ & $\mathrm{H}_{\gamma}$ & $5 \times 10^{31}$ & $8 \times 10^{32}-3 \times 10^{33}$ \\
\hline 2 & AT Mic & 600 & $10^{14}-10^{16}$ & $2-200 \times 10^{29}$ & $\mathrm{H}_{\delta}$ & $3 \times 10^{31}$ & $3 \times 10^{32}$ \\
\hline 3 & wTTs Cham & 600 & $1.4-78 \times 10^{18}$ & $2.5-140 \times 10^{33}$ & $\mathrm{H}_{\alpha}$ & $2 \times 10^{33}-10^{34}$ & $3.2 \times 10^{34}-1.6 \times 10^{35}$ \\
\hline 4 & AU Mic & 1400 & $10^{20}$ & $10^{36}$ & $\mathrm{BB}^{\mathrm{a}}$ & $3 \times 10^{35}$ & $\sim 3 \times 10^{34}$ \\
\hline 6 & DENIS 1048-39 & 100 & $3-30 \times 10^{16}$ & $1.5-15 \times 10^{30}$ & $\mathrm{H}_{\alpha}, \mathrm{H}_{\beta}$ & $9 \times 10^{29}$ & $9 \times 10^{30}$ \\
\hline 7 & AD Leo & 84 & $4-500 \times 10^{17}$ & $1.5-150 \times 10^{31}$ & C III & $2 \times 10^{31}$ & $2 \times 10^{32}$ \\
\hline 8 & V374 Peg & 675 & $10^{16}-10^{17}$ & $2-20 \times 10^{31}$ & $\mathrm{H}_{\alpha}$ & $\sim 10^{33}$ & $\sim 1.6 \times 10^{34}$ \\
\hline $\mathrm{X}$-rays & Star & $u_{\mathrm{CME}}\left(\mathrm{km} \mathrm{s}^{-1}\right)$ & $M_{\mathrm{CME}}(\mathrm{g})$ & $E_{k, \mathrm{CME}}(\mathrm{erg})$ & $\tau_{1 / 2}(\mathrm{ks})$ & $L\left(R_{\odot}\right)$ & $F_{\mathrm{X}}(\mathrm{erg})$ \\
\hline 9 & Algol $\mathrm{B}^{\mathrm{b}}$ & $250-6600$ & $2 \times 10^{21}-2 \times 10^{22}$ & $7 \times 10^{35}-3 \times 10^{38}$ & 5.6 & & $10^{37}$ \\
\hline 10 & Prox Cent & $40-1000$ & $1.2 \times 10^{16}-7 \times 10^{18}$ & $10^{29}-4 \times 10^{34}$ & 0.5 & $0.03-0.75$ & $1.7 \times 10^{31}$ \\
\hline 11 & Algol & $280-2400$ & $6 \times 10^{19}-4.5 \times 10^{21}$ & $2.3 \times 10^{34}-1.3 \times 10^{38}$ & 5 & $2-17.5$ & $1.15 \times 10^{37}$ \\
\hline 12 & V773 Tau & $210-730$ & $10^{20}-1.4 \times 10^{21}$ & $2.5 \times 10^{34}-3.7 \times 10^{36}$ & 20 & $6-21$ & $8.8 \times 10^{36}$ \\
\hline 13 & UX Arietis & & Prominence? & & & & $\geqslant 3.7 \times 10^{36}$ \\
\hline 14 & Pleiades & & Prominence? & & & & $8.3 \times 10^{33}$ \\
\hline 15 & $\sigma \mathrm{Gem}$ & $500-2800$ & $3 \times 10^{21}-10^{23}$ & $4 \times 10^{36}-4 \times 10^{39}$ & 12.5 & $9-51$ & $6.4 \times 10^{37}$ \\
\hline
\end{tabular}

Notes. The first five columns are the same observational methods. Column 1 indicates the original reference, column 2 the star observed, column 3 the derived CME speed, column 4 the derived CME mass, and column 5 the CME kinetic energy. Doppler shifts: column 6 indicates the emission passband, column 7 the total measured fluence, and column 8 the converted X-ray fluence. X-ray absorption: column 6 indicates the time for the column density to reach half its maximum value, column 7 the flaring loop length, and column 8 the X-ray fluences converted to the GOES passband (1-8 $\AA$ ) using the Chandra/PIMMS online tool and setting the plasma model to MEKAL, the galactic column density to $N_{\mathrm{H}}=10^{20} \mathrm{~cm}^{-2}$, and the abundance to 0.4 times the solar value.

a Broadband.

${ }^{\mathrm{b}}$ CME characteristics taken from Moschou et al. (2017).

References. Same as those in Tables 1 and 2.

CME, and finding that adiabatic expansion alone would give a slower flare decay than observed.

\section{Results}

Using the stellar CME candidates described above, we estimated the mass and kinetic energy of each event, assuming that they escaped the gravitational potential of the host star. Our results are presented in Table 3. For the events observed through Doppler shifts, we show the wave band in column 6 and the observed fluence in that wave band in column 7. Then, using the statistical relation (Equation (1)), we convert the observed fluences to soft X-ray fluence. The observed CME plasma speeds are presented in column 3 and the estimated masses in column 4. Based on these values, we estimate the CME kinetic energy (column 5) for the cases where this estimation was not performed on the original paper.

For the events observed through X-ray absorption, we have chosen an $e$-folding time (column 6 ) for the column density decay and two length scales (column 7), one obscuring (equal to the flaring loop size $L_{\mathrm{obs}}=L_{\text {flaring loop }}$ ) and one dynamic $\left(L_{\mathrm{dyn}}=5 R_{\star}\right)$. Based on these length and timescales, we then calculate the CME speed (column 3), mass (column 4), and kinetic energy (column 5). Column 8 indicates the $\mathrm{X}$-ray fluence for each case in the GOES passband (1-8 $)$. The conversions from each X-ray instrument to GOES fluences were performed using the MEKAL plasma model.

The analyzable CME candidates presented in Table 3 are plotted in Figure 2 together with typical solar events and historic energetic events. Our data size is not large (only 12 points), and the event characteristics have large errors due to the indirect way of inferring them. However, we can already draw some interesting conclusions.
CME masses. The top panel of Figure 2 shows the estimated stellar CME masses (present work and Moschou et al. 2017) with green square symbols, overplotted with solar events in gray squares from the Yashiro \& Gopalswamy (2009) compilation (see also Drake et al. 2013) and three historic energetic solar events in distinctly colored squares (Carrington and 775 AD events, Melott \& Thomas 2012; 660 BC event, O'Hare et al. 2019). The inferred characteristics of the stellar events appear to follow the extrapolated solar trend from Drake et al. (2013), albeit with a reasonably large spread of points and uncertainties in the stellar data. We return to this relation in Section 5.4 below.

CME kinetic energies. We illustrate in the bottom panel of Figure 2 the estimated stellar CME kinetic energies (present work and Moschou et al. 2017) with green triangles, overplotted with solar events in gray triangles (Yashiro \& Gopalswamy 2009; Drake et al. 2013) and historic energetic solar events in distinctly colored triangles (Carrington and 775 AD events, Melott \& Thomas 2012; 660 BC event, O'Hare et al. 2019). The kinetic energies of energetic stellar events appear to deviate from the extrapolated solar trend of Drake et al. (2013). This is consistent with the conclusions drawn in Drake et al. (2013) that the solar CME-flare relation is most probably breaking down in the very active stellar regime, and simple solar extrapolation overestimates the fraction of the bolometric energy that a stellar CME takes from an active star (see also Section 5.4 below).

\section{Discussion}

\subsection{Sources of Error and Discrepancy}

The analysis of historical CME candidates presented here necessarily involves a number of approximations and assumptions that inevitably lead to nonnegligible sources of systematic 
uncertainty. We discuss some aspects of these uncertainties below.

Emission from stellar flares and the emitting plasma characteristics inferred can be used to study stellar CMEs, assuming that the solar CME-flare relation can be extended in the stellar regime (e.g., Aarnio et al. 2012; Drake et al. 2013). However, flaring properties between the Sun and active stars may differ substantially. As Briggs \& Pye (2003) argued, the quiescent main mass of the solar coronal plasma emits X-rays corresponding to $1-2 \mathrm{MK}$ with flaring plasma reaching temperatures of the order of $10 \mathrm{MK}$. Active stars have coronae with plasma in the $20 \mathrm{MK}$ range and flaring plasma corresponding to $100 \mathrm{MK}$. In the solar corona, low first ionization potential (FIP) elements, e.g., $\mathrm{Mg}, \mathrm{Si}$, and $\mathrm{Fe}$, are generally overabundant in comparison to high-FIP elements, such as $\mathrm{O}$, $\mathrm{Ne}$, and Ar. In contrast, in very active stellar coronae, the pattern is reversed into an inverse FIP effect, with low-FIP elements appearing underabundant relative to high-FIP elements. These abundance fractionation patterns appear to be a function of both activity level and spectral type (see, e.g., Drake 2003; Robrade \& Schmitt 2005; Laming 2015; Wood et al. 2018). This is evidence that stellar CMEs could consist of plasma with different characteristics-both temperature and chemical composition - than the plasma in solar CMEs. In order to better comprehend the stellar CME-flare relation, it is important to combine both observational and computational work.

It is not a straightforward task to discriminate between escaping atmospheric material and photospheric evaporation. In most cases, spatial resolution is a problem when trying to locate stellar flares, which is important for flaring loop length estimations, since most stars appear as point sources from the Earth's orbit. More specifically, Covino et al. (2001) noted that most flaring loop size estimations have been performed based on theoretical models, which make assumptions for the heating during the decay phase (see, e.g., Reale et al. 1997 for a sustained heating), apart from the unique case of Favata \& Schmitt (1999), where the flare was pinpointed to originate from the south pole of Algol B. Covino et al. (2001) argued that errors can arise through this process, especially when heating is present during the flare decay phase. Things get even more complicated when we consider that oftentimes, there are two or more plasma temperature components present during the evolution of a flaring event (see, e.g., Covino et al. 2001).

It is not only the plasma temperature but also the metallicity and column density that will play a role in the relevant physics. When there is a high enough count rate and observed spectra can be time-resolved, one can fit the observations with plasma models and infer these quantities. For example, Covino et al. (2001) used different one-, two-, and three-temperature models to fit their observations and were able to derive a global coronal metallicity $Z$ for the active star Gl 355 (LQ Hya) that is of the order of $Z / Z \sim 0.1$, indicating that the corona is characterized by the inverse FIP effect referred to above. This serves as an extra pointer that the coronal conditions in active stars might differ substantially and that simple extrapolations from solar events should be treated with some caution.

\subsubsection{CMEs Inferred Using Doppler Shifts}

Blue- and redshift signals can arise from plasma motion that does not necessarily arise from escaping outflows that are associated with CMEs. In Figure 3, we illustrate the scenario of

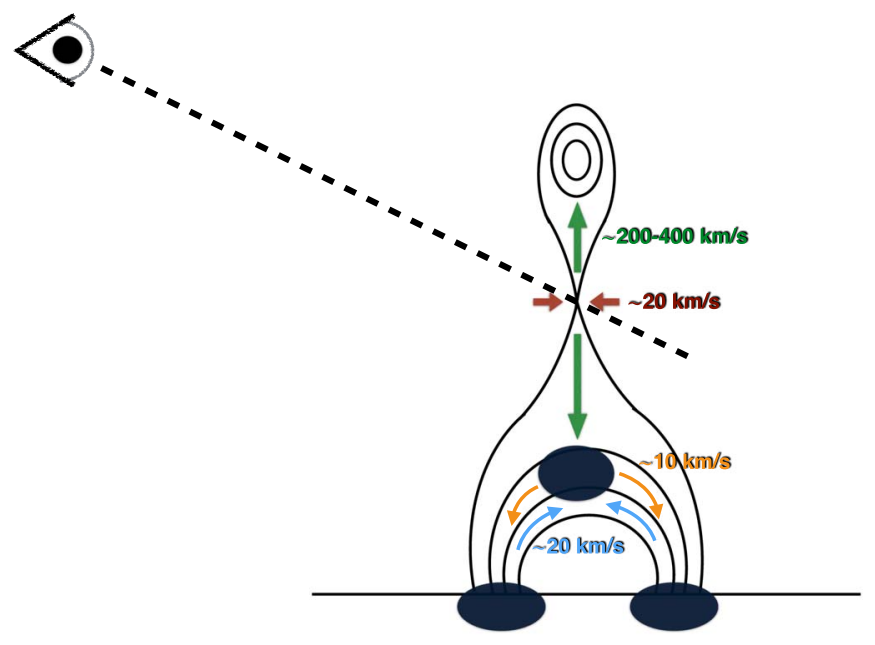

Figure 3. Schematic demonstrating the standard CME-flare scenario in the Sun and the multiple flows triggered by reconnection and the subsequent atmospheric response according to Hara et al. (2011). The blue blobs indicate the X-ray emission sources.

a reconnection-triggered flare and all the different up- and downflows generated as a result. Depending on the line profiles used to estimate the Doppler shift, different atmospheric layers are probed.

Blueshifts in the range of a few tens to a couple hundred $\mathrm{km} \mathrm{s}^{-1}$ that have often been reported can be confused with chromospheric evaporation, which is essentially the response of the chromosphere when accelerated particles from the corona collide with the dense local material and heat it up, causing evaporation. This evaporation is an upflow, but the material is generally contained within closed magnetic loops and does not escape.

Bopp \& Moffett (1973) estimated redshifts of the order of $1100 \mathrm{~km} \mathrm{~s}^{-1}$ in the Balmer lines and $600 \mathrm{~km} \mathrm{~s}^{-1}$ in the Ca II K line for UV Ceti. Strong redshifts might arise from material falling onto the stellar surface but could also be associated with CMEs that are traveling in the opposite hemisphere and direction to that facing the observer; see the second panel of Figure 4. Blueshift measurements of speeds larger than the local escape speed thus provide a more conclusive signature of material escaping the gravitational pull of the star than redshifts, which might or might not indicate escaping material.

As Houdebine et al. (1990) mentioned, projection effects can be very important and add an extra level of uncertainty in the measured speeds. More specifically, projection effects only allow for the determination of the lower limits of the true CME speeds, which could be severely underestimated if the CME propagation direction forms a large angle with the line of sight. This concept is demonstrated in the first panel of Figure 4.

Another point of confusion could arise from the fact that CMEs and monster CMEs from active stars (Moschou et al. 2017; Alvarado-Gómez et al. 2018) are large structures comprising an array of heterogeneous plasma elements that, in principle, could move in different directions, e.g., in halo CMEs or CMEs with large opening angles. As a result of this process, both blueshifts and redshifts could be observed from the same CME event.

\subsubsection{CMEs Inferred Using X-Rays}

Both noneruptive and eruptive prominences are observed frequently in the Sun. In a few stellar CME candidates (e.g., 

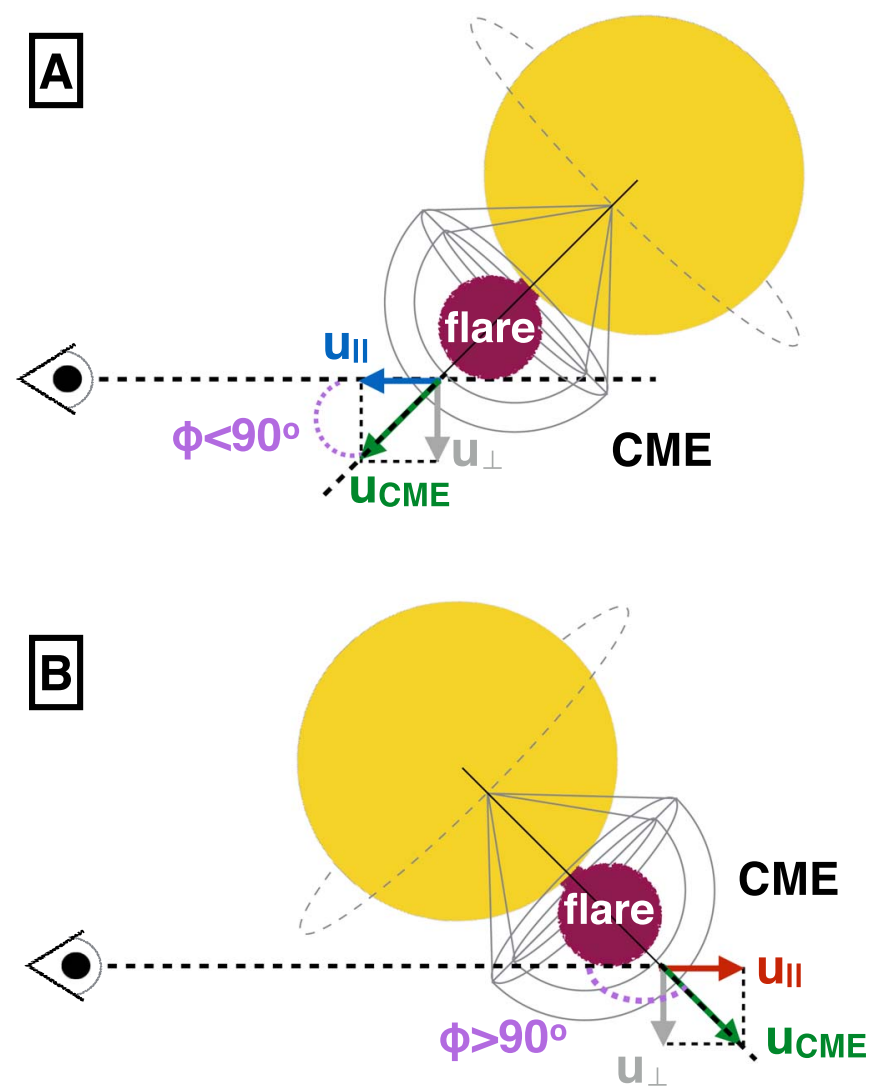

Figure 4. Schematic demonstrating the scenario of a CME expanding and propagating away from the star and giving rise to either blue- or redshift signatures as a result.

Ottmann \& Schmitt 1996), a substantial column density increase is observed, but there is no gradual decay seen during the observation. Those cases are likely not CME events but rather prominences that do not appear to erupt during the observation time. Gopalswamy et al. (2003) revealed a close relation between eruptive prominences and CMEs, determining an association rate of $83 \%$ using microwave data. However, the Gopalswamy et al. (2003) results contradict the poor association (10\%-30\%) found earlier by Wang \& Goode (1998) and Yang \& Wang (2002). More recently, Loboda \& Bogachev (2015) showed that most prominences (92\%) are stable and do not exhibit any apparent bulk motion. Furthermore, smaller prominences in their sample dating from between 2008 and 2009 appeared to be more dynamic than larger ones, with eruptive prominences following the same trends and the only difference being that they were larger. Thus, Loboda \& Bogachev (2015) concluded that there is a critical prominence mass beyond which further mass loading will lead to eruption. In other words, only massive enough prominences will erupt.

It is worth noting that most CME candidates found in X-ray observations had inferred loop flaring lengths much larger than those of solar events (Covino et al. 2001), oftentimes with semi-lengths of loops larger than the stellar radius. Furthermore, Covino et al. (2001) argued that large flaring loop sizes are required to account for the large flaring energy without unrealistically high magnetic fields. Covino et al. (2001) then went a step further and compared the estimation of flaring loop sizes between a hydrodynamic decay model accounting for a sustained heating often reported during the decay phase (Reale et al. 1997) and the order-of-magnitude estimation presented in
Pallavicini et al. (1990), only to conclude that the results in terms of flaring volume and densities were not too dissimilar (less than a factor of 2 difference in all length estimations).

Later on, Reale et al. (2005) used nanoflares to heat coronal loops in MHD models. Heat pulses produced due to nanoflares finally heat the loop up to $1-1.5 \mathrm{MK}$. More recently, Reale (2016) used MHD models to show that there are largeamplitude oscillations in flare light curves if the nanoflare heat pulse is faster than the sound-crossing time of the emitting loop. Reale (2016) explained that this takes place because there is not enough time for pressure equilibrium to be reached during the heating phase, and shock waves are formed. Based on the fact that these oscillations are characteristic and differ from classic MHD waves, Reale (2016) was able to develop a new diagnostic for observing nonflaring coronal loops in both the solar and stellar regimes.

\subsection{Other Nominal CME Observational Methods}

\subsubsection{Type II Radio Bursts}

The comparative rarity of the CME candidates investigated in this study - only 15 events from the last several decades-is a testament to the difficulty in detecting and observing them. Until synoptic observations of the X-ray sky can be made with a sensitivity approaching that of current observatories, the number of X-ray absorption CME candidates is going to remain very low. Similarly, continuous wide-field spectroscopic monitoring will be required to significantly increase the rate of acquisition of Doppler-shift events.

Type II radio bursts are instead the most promising observational method for CME tracking, due to their 1:1 association with CMEs in the solar case. More than $120 \mathrm{hr}$ of observations with the Very Large Array (VLA) ${ }^{7}$ have been invested in the search for stellar CMEs in active stars (Crosley et al. 2016; Crosley \& Osten 2018a, 2018b; Villadsen \& Hallinan 2018) without any Type II radio burst detection yet.

Scintillation of background radio sources could also be a potential method for observing stellar CMEs, as mentioned in Osten et al. (2017). Interplanetary scintillation has already been applied to solar CMEs (e.g., Manoharan 2010). Manoharan (2010) used measurements of scintillation for a large number of radio sources, based on which he was then able to reconstruct a three-dimensional view of a propagating CME event.

The near-future Square Kilometre Array will offer the potential to be able to detect large CMEs on nearby stars. Until the commissioning of that facility, we do not envisage that the stellar CME sample presented here will be greatly enlarged upon.

\subsubsection{EUV Dimmings}

In the solar case, when a CME erupts and propagates away from the Sun, it vacates the low-lying solar atmospheric material inside the CME base. This process leaves a wellobserved footprint in EUV wavelengths known as EUV dimming (see, e.g., Zhukov \& Auchère 2004; Mason et al. 2014; Chandra et al. 2016). The most widely adopted interpretation of the coronal dimming signature is due to plasma evacuation resulting from an escaping CME. However,

\footnotetext{
7 The VLA is an interferometer array using the combined views of its 27 antennas to mimic the view of a telescope as big across as the farthest distance between its antennas, i.e., 22 miles (https://public.nrao.edu/telescopes/vla/).
} 
it is also possible that the coronal material changes its temperature (thus becoming dimmer or darker in filtergrams such as the ones from $S D O / \mathrm{AIA}$ ) while largely remaining in its original volume.

Evidence of a stellar UV dimming event on EV Lacertae was reported by Ambruster et al. (1986). The IUE observed EV Lac over a time span of 9 days for $4 \mathrm{hr}$ day $^{-1}$. Ambruster et al. (1986) noted a $1.5 \mathrm{hr}$ dimming of some UV wavelengths. Specifically, prominent UV line fluxes (C IV and $\mathrm{Mg}$ II) dropped by a factor of $\sim 2$ for about $1.5 \mathrm{hr}$. Ambruster et al. (1986) favored the scenario of a large CME as an explanation of their results.

There is currently no EUV observing facility flying with which to investigate dimming events on stars other than the Sun. Coronal dimming on the Sun amounts to only a few percent of the total signal (Mason et al. 2016) and is aided by the spatial resolution of the solar disk. Detections of EUV dimming will be challenging for stars, and long, continuous observations will be needed should a future EUV-capable observatory become available.

Finally, Osten et al. (2017) noted that other potential methods to observe stellar CMEs could be (a) pre-flare "dips" like the one reported in Giampapa et al. (1982) for EQ Peg and (b) the observational effect of CMEs in their immediate stellar surroundings by, for example, sweeping debris disks away from the host star (see, e.g., Melis et al. 2012; Osten et al. 2013). Interestingly, Melis et al. (2012) estimated that a CME event with mass of the order of $\sim 10^{20} \mathrm{~g}$ (which is well within the CME masses we estimated for stellar events; see Figure 2) would suffice to remove debris disk material with mass $\sim 10^{21} \mathrm{~g}$.

\subsection{White-light versus X-Ray Energy Partition}

In the solar case, the white-light component of the flare dominates over the soft X-ray emission in the GOES passband (1-8 $\AA$ ) by a factor of approximately 100 (e.g., Kretzschmar 2011; Emslie et al. 2012). For both Sun-like and active stars, Butler (1993) and Martínez-Arnáiz et al. (2011) showed that the X-ray emission is tens of times larger than the flux in individual Balmer lines. More specifically, Martínez-Arnáiz et al. (2011) showed that for Sun-like stars and lower fluences, there is an equipartition between X-rays and Balmer line emission, while the $\mathrm{X}$-rays become dominant and 10 times larger in the higher energies. There is currently no extensive stellar statistical study associating the white light to X-ray emission. We can then hypothesize that if the Balmer-to-X-ray relation extends to the integrated white-light part of the spectrum, we expect that the energy emitted in X-rays for more active stars will increase to more than $1 \%$. However, Procházka et al. (2018), for example, used sophisticated particle beam models to investigate the energy partition during the solar flare event of 2014 June 11 and specifically understand the suppressed Balmer line emission. The authors showed that during the impulsive phase of the flare, only the $\mathrm{H}_{\alpha}$ line was in emission, while higher Balmer lines remained in absorption. This is an example of how complex the whole image can get once we get a closer look at the multiwavelength energy distribution in flares, even in the solar case.

\subsection{Flare versus CME Occurrence Frequency and the Nature of CMEs on Active Stars}

The standard paradigm for the study of stellar flares has been the temporal correspondence of the continuum optical emission and signatures in optical, radio, and X-ray wavelengths due to the collisions of accelerated particles on the lower atmosphere known as the "Neupert effect," first established by Neupert (1968). This picture is essentially that illustrated in Figure 3, which is also thought to give rise to CMEs. Were this always the case, the flare-CME correspondence would be straightforward, and the inference of CME occurrence could be reliably deduced from flare observations.

An apparent breakdown of the Neupert effect in stellar flares was found by Osten et al. (2005) in a multiwavelength study of flares on EV Lac. No observable X-ray enhancement was seen for salient $U$-band flares, and the reverse was also the case. There is indeed a history of observations of a large fraction of flares on the Sun that do not conform to the standard tworibbon flare model in which soft X-rays arise from material evaporated from the chromosphere by energetic particle beams (e.g., Feldman 1990; Veronig et al. 2002; Fletcher et al. 2011).

While solar observations do demonstrate a strong association rate between CMEs and flares (e.g., Vršnak et al. 2004; Gopalswamy et al. 2010), far from all solar flares are associated with CMEs. The association rate is observed to grow from a few percent for weak flares to $90 \%$ or more for strong (X-class) flares (Gopalswamy et al. 2009). Compagnino et al. (2017) showed that the CME-flare association rate also strongly depends on the temporal window considered between the onset of the flare and the identification of the CME. Depending on the duration of that window, the association rate can be significantly lower than 1:1, even for X-class solar flares.

In the stellar regime, while numerous stellar flares and superflares are observed in Sun-like and active stars, there is a clear observational discrepancy between stellar flares and CMEs: as we have emphasized here, stellar CMEs cannot be imaged directly with current technological capabilities, and their indirect detection is currently extremely difficult. Existing studies extrapolating flare-CME relations for the Sun have assumed that the flare-CME association rates are the same.

Aarnio et al. (2012) estimated stellar CME frequencies using solar extrapolations for T Tauri stars. Drake et al. (2013) used solar CME-flare relations and measured solar CME properties to estimate the mass and kinetic energy stripped away from stars as a function of X-ray activity level (also see this approach as applied by Osten \& Wolk 2015). Drake et al. (2013) noted that the inferred mass- and energy-loss rates were unrealistically high for the most active stars. Kinetic energy requirements were especially problematic, amounting to about $10 \%$ of the stellar bolometric energy output. Drake et al. (2013) concluded that either the relationships between solar CME mass and speed and flare X-ray energy must break down for the most active stars, or the flare-CME association rate must drop significantly below 1 .

The stellar CME results in Figure 2 present a potential way out of the Drake et al. (2013) quandary. As noted in Section 4, the stellar CME kinetic energies versus flare X-ray energies lie significantly below the extrapolated solar relation. The inferred energies of the stellar events are, in fact, either at or beneath the line of parity drawn at equal kinetic and X-ray energy and placed about a factor of 200 lower than the extrapolated relation. This result is consistent with the Vida et al. (2019) 
analysis, wherein scientists suggest that the event masses increase faster than the event speeds (see also Section 2 and Equation (3)). The implication is that the kinetic energies of large stellar CMEs are more than 2 orders of magnitude less energetic than extrapolation suggests. Instead of CMEs on the most active stars requiring $10 \%$ of the bolometric stellar energy output, these events suggest that less than $0.1 \%$ is needed, which is then comparable to the X-ray luminosity at activity saturation, $L_{\mathrm{X}} / L_{\mathrm{bol}}=10^{-3}$. In short, if our derived CME energies are correct, the problematic energetic requirements noted by Drake et al. (2013) are alleviated.

At the same time, our inferred CME masses are more or less in agreement with the Drake et al. (2013) extrapolation, and the total CME mass-loss estimates by those authors still hold. For the most active Sun-like star at a saturated $L_{\mathrm{X}} / L_{\mathrm{bol}}=10^{-3}$ activity level, the Drake et al. (2013) extrapolation implies a mass-loss rate of a few $10^{-10} M_{\odot} \mathrm{yr}^{-1}$, falling off with decreasing X-ray luminosity according to $\dot{M} \propto L_{\mathrm{X}}^{1.5}$. This can be compared with the current solar wind mass-loss rate of approximately $2 \times 10^{-14} M_{\odot} \mathrm{yr}^{-1}$ (e.g., Cohen 2011, and references therein).

The "kinetic energy deficit" of very large stellar CMEs compared with solar extrapolations indicates that it is the CME velocity that is limited at the very energetic extreme, rather than the mass. The velocity could be somewhat self-limiting in being related both to the large-scale magnetic field of the host star, which can act as a restraining term (e.g., Alvarado-Gómez et al. 2018), and to the magnetic field it carries and its interaction with the stellar wind that provides a drag force (Cargill 2004; Vršnak et al. 2004; Žic et al. 2015; Moschou et al. 2017). All of these retarding influences get stronger with increasing magnetic activity.

The other way out of the CME energy budget conundrum noted by Drake et al. (2013) is a substantial decrease in the CME-flare association rate, such that the majority of the flares we observe on active stars are not accompanied by CMEs. Indeed, the observational mismatch between CMEs and flares poses a fundamental question: whether the combination of very rare CME candidates and very common flares is purely due to observational bias, or rather, whether there could be a fundamental mechanism suppressing the CME escape in specific scenarios.

The scenario of a CME suppression mechanism was raised by Drake et al. (2016), who suggested that the strong overlying magnetic field in active stars might prevent CMEs from escaping. This was examined in more detail in a recently published computational study by Alvarado-Gómez et al. (2018) and was also discussed by Odert et al. (2017). AlvaradoGómez et al. (2018) demonstrated that a strong large-scale overlying magnetic field can suppress a CME eruption that has a poloidal magnetic flux less than twice the total flaring energy of the associated flare, i.e., $E_{k} \geqslant 2 F_{\text {total }}$, where $E_{k}$ is the CME kinetic energy and $F_{\text {total }}$ is the total flaring energy in the entire electromagnetic spectrum. For the solar case, an overlying magnetic field of $75 \mathrm{G}$ is able to suppress all of the currently observable solar CME events.

Our stellar CME sample cannot address the CME-flare association rate because of the enormous observational bias toward flares rather than CMEs. Such a step requires the advent of much more sensitive methods of CME detection.

\subsection{No Way Out for the Early Faint Sun Paradox}

Copious mass loss through CMEs is potentially of great importance to the "early faint Sun paradox," first noted by Sagan \& Mullen (1972). Those authors pointed out that the lower solar luminosity earlier in the history of the solar system implies global mean Earth temperatures below the freezing point of seawater until about $2.3 \mathrm{Gyr}$ ago, in contradiction with geological evidence for liquid oceans. An early Sun more massive by several percent that has since been lost through mass loss provides a potential solution (e.g., Guzik et al. 1987; Sackmann \& Boothroyd 2003). Unfortunately, the conclusions of Drake et al. (2013; see also Osten \& Wolk 2015) would still hold: the CME-driven mass-loss rate would be insufficient for the 2 Gyr old Sun to account for the luminosity deficiency. Nevertheless, it would be of considerable interest to examine the observable consequences of very high, $\sim 10^{-10} M_{\odot} \mathrm{yr}^{-1}$, mass-loss rates for the most active stars. This regime might prove the most promising for testing CME-flare relations for the combination of frequent flaring and highest flare energies.

\subsection{Relevance for Exoplanet Impact}

Understanding the effects of stellar transient events on exoplanetary atmospheres is not straightforward. In two computational studies, Cohen et al. (2011) and Cerenkov et al. (2017) simulated the interaction between CMEs and the magnetospheres of hot Jupiters. Cohen et al. (2011) found that the planetary atmosphere is mostly shielded from the transient, even for a planetary magnetic field as low as $1 \mathrm{G}$, but did not perform an atmospheric loss analysis. Cerenkov et al. (2017), however, suggested that the interaction of CMEs with the hotJupiter envelope would substantially increase the mass-loss rate, with faster CMEs causing a higher mass-loss rate. A limitation in CME velocity that our results suggest is interesting from the perspective of extrapolating solar CME speeds and the impact of energetic CMEs on planets.

In the compilation of CME data by Yashiro \& Gopalswamy (2009), the maximum deduced speed for a solar CME is about $3000 \mathrm{~km} \mathrm{~s}^{-1}$, and the maximum associated flare energy is approximately $10^{31} \mathrm{erg}$. The stellar events examined here reach nearly 7 orders of magnitude greater flare energy, and extending the Yashiro \& Gopalswamy (2009) CME speedflare energy relation this far would indicate that speeds of 10,000-100,000 $\mathrm{km} \mathrm{s}^{-1}$ should be reached. The stellar CME kinetic energies we infer instead indicate that CME speeds should not be greatly in excess of those observed in the solar system. In terms of planetary impact, the ram pressure for an energetic stellar CME with density $\rho$ and moving at speed $v$, $\rho v^{2}$, is also expected to be 2 orders of magnitude lower than inferred from extrapolating solar data.

For a dipolar planetary magnetic field, the magnetospheric standoff distance at the equator, $r_{M}$, scales with wind (or CME) ram pressure, $P_{\text {ram }}$, as $r_{M} \propto P_{\text {ram }}^{-1 / 6}$ (Schield 1969; Gombosi 2004) such that a lower pressure by a factor of 100 implies a larger magnetosphere by a factor of $\sim 2$. The implication is that the ability of CMEs to dynamically strip planetary atmospheres is much reduced compared with CME energies inferred from the Drake et al. (2013) extrapolation.

\section{Conclusions}

We have presented a comprehensive study of currently known historic stellar CME candidates in the literature, 
commenting on their relative merits in each case. We analyzed each case separately to infer the CME mass and kinetic energy in order to better understand what historic events reveal about the stellar CME-flare relation and to examine similarities and differences with solar events. While the analysis necessarily requires some assumptions regarding CME geometries, and the resulting uncertainties for individual events can exceed an order of magnitude, the large dynamic range of CME and flare properties allows useful comparisons with solar events to be made.

The investigated CME candidates were observed using two methods, namely, continuous X-ray absorption and Doppler shifts. Our sample of 12 events indicates that energetic stellar CMEs appear to follow the average relation between solar CME mass and X-ray flaring energy. In contrast, stellar CME energies appear to have 200 times less kinetic energy than the solar extrapolation predicts. This latter result alleviates the problematic energy requirements that otherwise result if observed stellar flares are accompanied by CMEs with kinetic energies that follow the solar extrapolation.

The kinetic energies and masses we infer for energetic stellar events indicate that CME velocities are probably limited by retardation in the large-scale stellar magnetic field and drag in the stellar wind. Lower resulting CME kinetic energies present a much more optimistic scenario for planetary atmospheres in close proximity to active host stars, such as on planets in the habitable zones of $\mathrm{M}$ dwarfs.

We thank the anonymous referee for valuable comments and suggestions. S.P.M. was supported by NASA Living with a Star grant No. NNX16AC11G. J.J.D. was funded by NASA contract NAS8-03060 to the Chandra X-ray Center and thanks the director, Belinda Wilkes, for continuing advice and support. O.C. was supported by NASA Astrobiology Institute grant NNX15AE05G. J.D.A.G. was supported by Chandra grants AR4-15000X and GO5-16021X. C.G. was supported by SI Grand Challenges grant "Lessons from Mars: Are Habitable Atmospheres on Planets around M Dwarfs Viable?"

\section{ORCID iDs}

Sofia-Paraskevi Moschou (1) https://orcid.org/0000-00022470-2109

Jeremy J. Drake (ㄷ) https://orcid.org/0000-0002-0210-2276

Ofer Cohen (10 https://orcid.org/0000-0003-3721-0215

Julián D. Alvarado-Gómez (i) https://orcid.org/0000-00015052-3473

Cecilia Garraffo (i) https://orcid.org/0000-0002-8791-6286

Federico Fraschetti (i) https://orcid.org/0000-0002-5456-4771

\section{References}

Aarnio, A. N., Matt, S. P., \& Stassun, K. G. 2012, ApJ, 760, 9

Aarnio, A. N., Stassun, K. G., Hughes, W. J., \& McGregor, S. L. 2011, SoPh, 268, 195

Alvarado-Gómez, J. D., Drake, J. J., Cohen, O., Moschou, S. P., \& Garraffo, C. 2018, ApJ, 862, 93

Ambruster, C. W., Pettersen, B. R., Hawley, S., Coleman, L. A., \& Sandmann, W. H. 1986, in New Insights in Astrophysics. Eight Years of UV Astronomy with IUE 263, ed. E. J. Rolfe \& R. Wilson (Noordwijk: ESA), 137

Antonucci, E., Gabriel, A. H., Acton, L. W., et al. 1982, SoPh, 78, 107

Audard, M., Güdel, M., Drake, J. J., \& Kashyap, V. L. 2000, ApJ, 541, 396

Ballester, J. L., Carbonell, M., Soler, R., \& Terradas, J. 2018, A\&A, 609, A6 Bond, H. E., Mullan, D. J., O’Brien, M. S., \& Sion, E. M. 2001, ApJ, 560, 919 Bopp, B. W., \& Moffett, T. J. 1973, ApJ, 185, 239
Briggs, K. R., \& Pye, J. P. 2003, MNRAS, 345, 714

Butler, C. J. 1993, A\&A, 272, 507

Butler, C. J., Rodono, M., \& Foing, B. H. 1988, A\&A, 206, L1

Cane, H. V., Richardson, I. G., \& von Rosenvinge, T. T. 2010, JGRA, 115, A08101

Caramazza, M., Flaccomio, E., Micela, G., et al. 2007, A\&A, 471, 645

Cargill, P. J. 2004, SoPh, 221, 135

Cerenkov, A., Bisikalo, D., Fossati, L., \& Möstl, C. 2017, ApJ, 846, 31

Chandra, R., Chen, P. F., Fulara, A., Srivastava, A. K., \& Uddin, W. 2016, ApJ, 822, 106

Chandra, R., Filippov, B., Joshi, R., \& Schmieder, B. 2017, SoPh, 292, 81

Christian, D. J., Mathioudakis, M., Bloomfield, D. S., et al. 2006, A\&A, 454,889

Cliver, E. W., \& Dietrich, W. F. 2013, JSWSC, 3, A31

Cliver, E. W., Tylka, A. J., Dietrich, W. F., \& Ling, A. G. 2014, ApJ, 781, 32 Cohen, O. 2011, MNRAS, 417, 2592

Cohen, O., Kashyap, V. L., Drake, J. J., Sokolov, I. V., \& Gombosi, T. I. 2011, ApJ, 738, 166

Compagnino, A., Romano, P., \& Zuccarello, F. 2017, SoPh, 292, 5

Covino, S., Panzera, M. R., Tagliaferri, G., \& Pallavicini, R. 2001, A\&A, 371, 973

Cranmer, S. R. 2017, ApJ, 840, 114

Crosley, M. K., \& Osten, R. A. 2018a, ApJ, 856, 39

Crosley, M. K., \& Osten, R. A. 2018b, ApJ, 862, 113

Crosley, M. K., Osten, R. A., Broderick, J. W., et al. 2016, ApJ, 830, 24

Cully, S. L., Fisher, G. H., Abbott, M. J., \& Siegmund, O. H. W. 1994, ApJ, 435, 449

Daniel, K. J., Linsky, J. L., \& Gagné, M. 2002, ApJ, 578, 486

Drake, J. J. 2003, ApJ, 594, 496

Drake, J. J., Cohen, O., Garraffo, C., \& Kashyap, V. 2016, in IAU Symp. 320, Solar and Stellar Flares and their Effects on Planets, ed. A. G. Kosovichev, S. L. Hawley, \& P. Heinzel (Cambridge: Cambridge Univ. Press), 196

Drake, J. J., Cohen, O., Yashiro, S., \& Gopalswamy, N. 2013, ApJ, 764, 170 Emslie, A. G., Dennis, B. R., Shih, A. Y., et al. 2012, ApJ, 759, 71

Favata, F., \& Schmitt, J. H. M. M. 1999, A\&A, 350, 900

Feigelson, E. D., Hornschemeier, A. E., Micela, G., et al. 2004, ApJ, 611, 1107

Feldman, U. 1990, ApJ, 364, 322

Fisher, R. R., \& Munro, R. H. 1984, ApJ, 280, 428

Fletcher, L., Dennis, B. R., Hudson, H. S., et al. 2011, SSRv, 159, 19

Franciosini, E., Pallavicini, R., \& Tagliaferri, G. 2001, A\&A, 375, 196

Fuhrmeister, B., \& Schmitt, J. H. M. M. 2004, A\&A, 420, 1079

Giampapa, M. S., Africano, J. L., Klimke, A., et al. 1982, ApJL, 252, L39

Gombosi, T. I. 2004, Physics of the Space Environment (Cambridge: Cambridge Univ. Press)

Gopalswamy, N. 2016, GSL, 3, 8

Gopalswamy, N., Akiyama, S., \& Yashiro, S. 2009, in IAU Symp. 257, Universal Heliophysical Processes, ed. N. Gopalswamy \& D. F. Webb (Cambridge: Cambridge Univ. Press), 283

Gopalswamy, N., Shimojo, M., Lu, W., et al. 2003, ApJ, 586, 562

Gopalswamy, N., Yashiro, S., Michalek, G., et al. 2010, SunGe, 5, 7

Gosain, S., Filippov, B., Ajor Maurya, R., \& Chandra, R. 2016, ApJ, 821, 85 Graffagnino, V. G., Wonnacott, D., \& Schaeidt, S. 1995, MNRAS, 275, 129

Grosso, N., Montmerle, T., Feigelson, E. D., et al. 1997, Natur, 387, 56

Güdel, M., Linsky, J. L., Brown, A., \& Nagase, F. 1999, ApJ, 511, 405

Güdel, M., \& Nazé, Y. 2009, A\&ARv, 17, 309

Guenther, E. W., \& Emerson, J. P. 1997, A\&A, 321, 803

Guinan, E. F., \& Sion, E. M. 1984, AJ, 89, 1252

Gunn, A. G., Doyle, J. G., Mathioudakis, M., Houdebine, E. R., \& Avgoloupis, S. 1994, A\&A, 285, 489

Gupta, G. R., Sarkar, A., \& Tripathi, D. 2018, ApJ, 857, 137

Guzik, J. A., Willson, L. A., \& Brunish, W. M. 1987, ApJ, 319, 957

Haisch, B. M., Linsky, J. L., Bornmann, P. L., et al. 1983, ApJ, 267, 280

Hara, H., Watanabe, T., Harra, L. K., Culhane, J. L., \& Young, P. R. 2011, ApJ, 741, 107

Hertzsprung, E. 1947, AnLei, 19, A1

Houdebine, E. R., Foing, B. H., \& Rodono, M. 1990, A\&A, 238, 249

Howard, R. A., Michels, D. J., Sheeley, N. R., Jr., \& Koomen, M. J. 1982, ApJL, 263, L101

Huenemoerder, D. P., Schulz, N. S., Testa, P., et al. 2010, ApJ, 723, 1558

Hummel, C. A., Monnier, J. D., Roettenbacher, R. M., et al. 2017, ApJ, 844,115

Isobe, T., Feigelson, E. D., Akritas, M. G., \& Babu, G. J. 1990, ApJ, 364, 104 Jiang, Y.-C., Li, L.-P., \& Yang, L.-H. 2006, ChJAA, 6, 345

Joshi, N. C., Srivastava, A. K., Filippov, B., et al. 2013, ApJ, 771, 65

Kahler, S. W. 2001, JGR, 106, 20947

Kamata, Y., Koyama, K., Tsuboi, Y., \& Yamauchi, S. 1997, PASJ, 49, 461 
Kashyap, V. L., Drake, J. J., Güdel, M., \& Audard, M. 2002, ApJ, 580, 1118 Katsova, M. M., Drake, J. J., \& Livshits, M. A. 1999, ApJ, 510, 986

Kay, C., Opher, M., \& Kornbleuth, M. 2016, ApJ, 826, 195

Kervella, P., Thévenin, F., \& Lovis, C. 2017, A\&A, 598, L7

Khodachenko, M. L., Lammer, H., Lichtenegger, H. I. M., et al. 2007a, P\&SS, 55,631

Khodachenko, M. L., Ribas, I., Lammer, H., et al. 2007b, AsBio, 7, 167

Kirk, M. S., Balasubramaniam, K. S., Jackiewicz, J., \& Gilbert, H. R. 2017, in IAU Symp. 327, Fine Structure and Dynamics of the Solar Atmosphere (Cambridge: Cambridge Univ. Press), 117

Kopp, R. A., \& Poletto, G. 1984, SoPh, 93, 351

Kretzschmar, M. 2011, A\&A, 530, A84

Kundu, M. R., White, S. M., Garaimov, V. I., et al. 2004, ApJ, 607, 530

Laming, J. M. 2015, LRSP, 12, 2

Leitzinger, M., Odert, P., Greimel, R., et al. 2014, MNRAS, 443, 898

Leitzinger, M., Odert, P., Ribas, I., et al. 2011, A\&A, 536, A62

Loboda, I. P., \& Bogachev, S. A. 2015, SoPh, 290, 1963

Manoharan, P. K. 2010, SoPh, 265, 137

Martínez-Arnáiz, R., López-Santiago, J., Crespo- Chacón, I., \& Montes, D. 2011, MNRAS, 414, 2629

Mason, J. P., Woods, T. N., Caspi, A., Thompson, B. J., \& Hock, R. A. 2014, ApJ, 789, 61

Mason, J. P., Woods, T. N., Webb, D. F., et al. 2016, ApJ, 830, 20

Melis, C., Zuckerman, B., Rhee, J. H., et al. 2012, Natur, 487, 74

Melott, A. L., \& Thomas, B. C. 2012, Natur, 491, E1

Mewe, R., Kaastra, J. S., \& Liedahl, D. A. 1995, Legacy, 6, 16

Micela, G., Sciortino, S., Vaiana, G. S., et al. 1990, ApJ, 348, 557

Miyake, F., Nagaya, K., Masuda, K., \& Nakamura, T. 2012, Natur, 486, 240

Montmerle, T., Koch-Miramond, L., Falgarone, E., \& Grindlay, J. E. 1983, ApJ, 269, 182

Moschou, S. P., Drake, J. J., Cohen, O., Alvarado-Gomez, J. D., \& Garraffo, C. 2017, ApJ, 850, 191

Moschou, S. P., Keppens, R., Xia, C., \& Fang, X. 2015, AdSpR, 56, 2738

Neupert, W. M. 1968, ApJL, 153, L59

Nordon, R., Behar, E., \& Güdel, M. 2006, A\&A, 446, 621

Notsu, Y., Maehara, H., Shibayama, T., et al. 2016, 19th Cambridge Workshop on Cool Stars, Stellar Systems, and the Sun (CS19), 119

Ochsenbein, F., Bauer, P., \& Marcout, J. 2000, A\&AS, 143, 23

Odert, P., Leitzinger, M., Hanslmeier, A., \& Lammer, H. 2017, MNRAS, 472,876

O’Hare, P., Mekhaldi, F., Adolphi, F., et al. 2019, PNAS, 116, 5961

Osten, R., Livio, M., Lubow, S., et al. 2013, ApJL, 765, L44

Osten, R. A., Hawley, S. L., Allred, J. C., Johns-Krull, C. M., \& Roark, C. 2005, ApJ, 621, 398

Osten, R. A., \& Wolk, S. J. 2015, ApJ, 809, 79

Osten, R. A., Wolk, S. J., Nandy, D., Valio, A., \& Petit, P. 2017, in IAU Symp. 328, Living Around Active Stars 328 (Cambridge: Cambridge Univ. Press), 243

Ottmann, R. 1994, A\&A, 286, L27

Ottmann, R., \& Schmitt, J. H. M. M. 1996, A\&A, 307, 813

Pallavicini, R., Serio, S., \& Vaiana, G. S. 1977, ApJ, 216, 108

Pallavicini, R., Tagliaferri, G., \& Stella, L. 1990, A\&A, 228, 403

Pandey, J. C., \& Singh, K. P. 2008, MNRAS, 387, 1627

Pandey, J. C., \& Singh, K. P. 2012, MNRAS, 419, 1219

Paresce, F. 1984, AJ, 89, 1022

Phillips, R. B., Lonsdale, C. J., \& Feigelson, E. D. 1991, ApJ, 382, 261

Poletto, G., Pallavicini, R., \& Kopp, R. A. 1988, A\&A, 201, 93

Preibisch, T., Neuhaeuser, R., \& Alcala, J. M. 1995, A\&A, 304, L13

Procházka, O., Reid, A., Milligan, R. O., et al. 2018, ApJ, 862, 76

Raymond, J. C., \& Smith, B. W. 1977, ApJS, 35, 419
Reale, F. 2007, A\&A, 471, 271

Reale, F. 2016, ApJL, 826, L20

Reale, F., Betta, R., Peres, G., Serio, S., \& McTiernan, J. 1997, A\&A, 325, 782

Reale, F., Güdel, M., Peres, G., \& Audard, M. 2004, A\&A, 416, 733

Reale, F., Nigro, G., Malara, F., Peres, G., \& Veltri, P. 2005, ApJ, 633, 489

Reames, D. V. 2013, SSRv, 175, 53

Richards, M. T. 1993, ApJS, 86, 255

Robrade, J., \& Schmitt, J. H. M. M. 2005, A\&A, 435, 1073

Roettenbacher, R. M., Monnier, J. D., Henry, G. W., et al. 2015, ApJ, 807, 23

Sackmann, I. J., \& Boothroyd, A. I. 2003, ApJ, 583, 1024

Sagan, C., \& Mullen, G. 1972, Sci, 177, 52

Schield, M. A. 1969, JGR, 74, 1275

Schmitt, J. H. M. M. 1994, ApJS, 90, 735

Schmitt, J. H. M. M., \& Liefke, C. 2004, A\&A, 417, 651

Shibata, K., \& Magara, T. 2011, LRSP, 8, 6

Skinner, S. L. 1993, ApJ, 408, 660

Smith, R. K., Brickhouse, N. S., Liedahl, D. A., \& Raymond, J. C. 2001, ApJL 556, L91

Somov, B. 1992, ass1, 172

Stelzer, B., \& Neuhäuser, R. 2001, A\&A, 377, 538

Subramanian, P., \& Dere, K. P. 2001, ApJ, 561, 372

Telleschi, A., Güdel, M., Briggs, K. R., Audard, M., \& Palla, F. 2007, A\&A, 468, 425

Teriaca, L., Falchi, A., Cauzzi, G., et al. 2003, ApJ, 588, 596

Tousey, R., Bartoe, J. D. F., Bohlin, J. D., et al. 1973, SoPh, 33, 265

Tsuboi, Y., Imanishi, K., Koyama, K., Grosso, N., \& Montmerle, T. 2000, ApJ, 532, 1089

Tsuboi, Y., Koyama, K., Murakami, H., et al. 1998, ApJ, 503, 894

Vemareddy, P., Maurya, R. A., \& Ambastha, A. 2012, SoPh, 277, 337

Veronig, A., Vršnak, B., Dennis, B. R., et al. 2002, A\&A, 392, 699

Vida, K., Kriskovics, L., Oláh, K., et al. 2016, A\&A, 590, A11

Vida, K., Leitzinger, M., Kriskovics, L., et al. 2019, A\&A, 623, A49

Villadsen, J., \& Hallinan, G. 2019, ApJ, 871, 214

Vourlidas, A., Howard, R. A., Esfandiari, E., et al. 2011, ApJ, 730, 59

Vršnak, B., Ruždjak, D., Sudar, D., \& Gopalswamy, N. 2004, A\&A, 423, 717

Wang, H., \& Goode, P. R. 1998, in ASP Conf. Ser. 140, Synoptic Solar Physics, ed. K. S. Balasubramaniam, J. Harvey, \& D. Rabin (San Francisco, CA: ASP), 497

Webb, D. F., Howard, T. A., Fry, C. D., et al. 2009, SpWea, 7, S05002

Welty, A. D. 1995, AJ, 110, 776

White, S. M., Pallavicini, R., \& Kundu, M. R. 1992, A\&A, 257, 557

Wood, B. E., Laming, J. M., Warren, H. P., \& Poppenhaeger, K. 2018, ApJ, 862,66

Wood, B. E., Redfield, S., Linsky, J. L., Müller, H.-R., \& Zank, G. P. 2005, ApJS, 159, 118

Wright, N. J., Drake, J. J., Mamajek, E. E., \& Henry, G. W. 2011, ApJ, 743, 48

Wright, N. J., Newton, E. R., Williams, P. K. G., Drake, J. J., \& Yadav, R. K. 2018, MNRAS, 479, 2351

Xie, H., Ofman, L., \& Lawrence, G. 2004, JGRA, 109, A03109

Yang, G., \& Wang, H. 2002, in Solar-Terrestrial Magnetic Activity and Space Environment, ed. H. Wang \& R. Xu (Boston, MA: Pergamon Press), 113

Yashiro, S., \& Gopalswamy, N. 2009, in IAU Symp. 257, Universal Heliophysical Processes, ed. N. Gopalswamy \& D. F. Webb (Cambridge: Cambridge Univ. Press), 233

Zhang, J., Richardson, I. G., Webb, D. F., et al. 2007, JGRA, 112, A10102

Zhao, X. P., Plunkett, S. P., \& Liu, W. 2002, JGRA, 107, 1223

Zhukov, A. N., \& Auchère, F. 2004, A\&A, 427, 705

Žic, T., Vršnak, B., \& Temmer, M. 2015, ApJS, 218, 32

Zuccarello, F. P., Chandra, R., Schmieder, B., Aulanier, G., \& Joshi, R. 2017, A\&A, 601, A26 